\title{
Berbamine Analogs Exhibit Differential Protective Effects From Aminoglycoside-Induced Hair Cell Death
}

\author{
Alexandria M. Hudson ${ }^{1}$, Gavin M. Lockard ${ }^{2 \dagger}$, Ojas A. Namjoshi ${ }^{3}$, Joseph W. Wilson ${ }^{3}$, \\ Katie S. Kindt ${ }^{4}$, Bruce E. Blough ${ }^{3}$ and Allison B. Coffin ${ }^{1,2 *}$ \\ ${ }^{1}$ Integrative Physiology and Neuroscience, Washington State University, Vancouver, WA, United States, ${ }^{2}$ College of Arts and \\ Sciences, Washington State University, Vancouver, WA, United States, ${ }^{3}$ RTI International, Research Triangle Park, NC, \\ United States, ${ }^{4}$ National Institute on Deafness and Other Communication Disorders, National Institutes of Health, Bethesda, \\ MD, United States
}

OPEN ACCESS

Edited by:

Michael E. Smith,

Western Kentucky University,

United States

Reviewed by:

Alan G. Cheng,

Stanford University, United States

Emma J. Kenyon,

University of Sussex, United Kingdom

*Correspondence:

Allison B. Coffin

allison.coffin@wsu.edu

${ }^{\dagger}$ Present address:

Gavin M. Lockard,

College of Pharmacy, Washington

State University, Spokane, WA,

United States

Specialty section:

This article was submitted to Cellular Neuropathology, a section of the journal

Frontiers in Cellular Neuroscience

Received: 09 April 2020 Accepted: 02 July 2020

Published: 29 July 2020

Citation:

Hudson AM, Lockard GM, Namjoshi OA, Wilson JW, Kindt KS, Blough $B E$ and Coffin $A B$ (2020) Berbamine Analogs Exhibit Differential Protective Effects From Aminoglycoside-Induced Hair Cell Death.

Front. Cell. Neurosci. 14:234. doi: 10.3389/fncel.2020.00234
Hearing loss is the third most common chronic health condition in the United States and largely results from damage to sensory hair cells. Major causes of hair cell damage include aging, noise exposure, and medications such as aminoglycoside antibiotics. Due to their potent antibacterial properties and low cost, aminoglycosides are often used for the treatment of gram-negative bacterial infections, surpassing expensive antibiotics with fewer harmful side effects. However, their use is coupled with permanent hearing loss in over $20 \%$ of patients requiring these life-sustaining antibiotics. There are currently no FDA-approved drugs that prevent hearing loss from aminoglycosides. A previous study by our group identified the plant alkaloid berbamine as a strong protectant of zebrafish lateral line hair cells from aminoglycoside damage. This effect is likely due to a block of the mechanotransduction channel, thereby reducing aminoglycoside entry into hair cells. The present study builds on this previous work, investigating 16 synthetic berbamine analogs to determine the core structure underlying their protective mechanisms. We demonstrate that nearly all of these berbamine analogs robustly protect lateral line hair cells from ototoxic damage, with $\mathrm{ED}_{50}$ values nearing $20 \mathrm{nM}$ for the most potent analogs. Of the 16 analogs tested, nine strongly protected hair cells from both neomycin and gentamicin damage, while one conferred strong protection only from gentamicin. These data are consistent with prior research demonstrating that different aminoglycosides activate somewhat distinct mechanisms of damage. Regardless of the mechanism, protection required the entire berbamine scaffold. Phenolic alkylation or acylation with lipophilic groups appeared to improve protection compared to berbamine, implying that these structures may be responsible for mitigating damage. While the majority of analogs confer protection by blocking aminoglycoside uptake, $18 \%$ of our analogs also confer protection via an uptake-independent mechanism; these analogs

Abbreviations: MET, mechanotransduction; ${ }^{1} \mathrm{H}$ NMR, nuclear magnetic resonance spectra for proton; TLC, thin-layer chromatography; EM, E2 embryo medium; dpf, days post-fertilization; DMSO, dimethylsulfoxide; DASPEI, 2-[4(dimethylamino)styryl]-N-ethylpyridinium iodide; MS-222, Tricaine methanesulfonate; GTTR, gentamicin conjugated with Texas Red; OPC, optimal protective concentration; TRP channels, transient receptor potential channels; NMDA, N-methyl$\mathrm{D}$-aspartate; AMPA, $\alpha$-amino-3-hydroxy-5-methyl-4-isoxazolepropionic acid; NF- $\mathrm{kB}$, nuclear factor $\kappa$-light-chain-enhancer of activated B cells; JNK, c-Jun N-terminal kinase pathways; ERK, extracellular receptor kinase. 
exhibited protection when delivered after aminoglycoside removal. Based on our studies, berbamine analogs represent a promising tool to further understand the pathology of aminoglycoside-induced hearing loss and can serve as lead compounds to develop otoprotective drugs.

Keywords: hair cell, aminoglycoside, zebrafish, lateral line, berbamine, ototoxicity, hearing loss, mechanotransduction

\section{INTRODUCTION}

Sensory hair cells can be damaged from aging, intense noise exposure, and medications such as aminoglycoside antibiotics. In mammals, damaged hair cells are not replaced, which can result in permanent hearing loss. In humans, hearing loss can be devastating since it can lead to social isolation and decreased employment opportunities (Raviv et al., 2010; Jung and Bhattacharyya, 2012; Bainbridge and Wallhagen, 2014; Mick et al., 2014). Despite the adverse effects of aminoglycosides on hearing, they are widely used in developing nations due to their potent antibacterial properties and low cost, surpassing expensive antibiotics with fewer harmful side effects. Currently, there are no FDA-approved drugs that prevent aminoglycosideinduced hearing loss. Our work seeks to develop a drug therapy that robustly prevents aminoglycoside-induced hearing loss by modifying the chemical scaffold of a naturally occurring otoprotective compound.

There is strong evidence supporting mechanoelectrical transduction (MET) as the primary path for aminoglycosides to enter hair cells. The MET channels in hair cells are non-selective cation channels with a pore diameter of $1.25 \mathrm{~nm}$. These features enable relatively large positively charged aminoglycosides to permeate the channel (Farris et al., 2004). Additionally, there is a membrane potential difference between the extracellular fluid and the negatively polarized cytoplasm that increases cellular uptake of positively charged aminoglycosides (Marcotti et al., 2005; Myrdal and Steyger, 2005). Pharmacologically blocking the MET channel dramatically decreases aminoglycoside uptake (Marcotti et al., 2005) further supporting antibiotic entry via this route. In addition to MET channels, there are also secondary entry routes occurring via endocytosis or through other ion channels (Portmann et al., 1974; Myrdal and Steyger, 2005; Karasawa et al., 2008; Hailey et al., 2017). The current hypothesis surrounding entry via endocytosis is that aminoglycosides are initially sequestered by endosomes, then trafficked to lysosomes, but different aminoglycosides (e.g., neomycin vs. gentamicin) differ in their rates of uptake into subcellular compartments. These data imply that sequestration of aminoglycosides in lysosomes could potentially attenuate hair cell damage (Hailey et al., 2017). Regardless of the entry route, aminoglycosides accumulate in hair cells, leading to pathological consequences.

In light of our understanding of the mechanisms of aminoglycoside toxicity, new targets for protection are arising. Given that the MET channel is the primary entry route for aminoglycosides, one option for protection is to block entry of aminoglycosides through the channel. Prior work using a zebrafish lateral line assay identified two such compounds,
PROTO-1 and PROTO-2, both of which protected hair cells from neomycin toxicity (Owens et al., 2008). Optimization of PROTO-1 yielded ORC-13661, an otoprotective lead compound that acts as a permeant MET channel blocker (Owens et al., 2008; Chowdhury et al., 2018; Kitcher et al., 2019). In a separate study, Kenyon et al. (2017) used zebrafish to identify an N-methyl-Daspartate (NMDA) receptor antagonist and a selective potassium channel antagonist that also protected hair cells by attenuating aminoglycoside entry. Here, we use a zebrafish lateral line assay to assess the relative protection conferred from a modified scaffold of an otoprotective plant alkaloid. Our modifications are designed to diversify the alkaloid's pharmacological activity to modulate multiple aspects of hair cell death, leading to a stronger therapy.

A previous study by our lab screened 502 natural compounds using a zebrafish model for ototoxicity and identified four otoprotective bisbenzylisoquinoline analogs: berbamine, E6 berbamine, hernandezine, and isotetrandrine, with berbamine being the most protective (Kruger et al., 2016). These analogs share a macrocyclic bistetrahydroisoquinoline ring scaffold and robustly protect hair cells from aminoglycoside damage, likely by attenuating aminoglycoside entry. These data are consistent with Ou et al. (2009, 2012), who demonstrated that quinoline ring compounds such as tacrine and chloroquine reduce aminoglycoside uptake by hair cells, leading to increased hair cell survival. Berbamine also reduces aminoglycoside-induced hair cell death in mice, likely by reducing aminoglycoside loading into the cochlea (Kirkwood et al., 2017). However, high concentrations of berbamine $(30 \mu \mathrm{M})$ were toxic to murine cochlear hair cells. Screening additional berbamine analogs offer an excellent opportunity to identify moieties that are responsible for berbamine's protective activity while avoiding the toxicity seen at high concentrations. This information will allow us to develop a non-toxic compound that maintains the diverse and protective pharmacological properties of berbamine to develop a robust therapeutic compound.

Bisbenzylisoquinoline compounds have recently attracted attention as therapeutics due to their innate chemical diversity and wide-ranging pharmacological effects, making them powerful scaffolds for drug optimization (Tian and Zheng, 2017; Teles et al., 2019). Berbamine, a plant alkaloid derived from Berberidaceae (barberry plants), has its pharmacological origins in traditional Chinese medicine as an anti-inflammatory agent. More recently, berbamine has shown potential as a therapeutic to reduce inflammation from a diverse array of cancers, thus inhibiting tumor cell invasion (Ren et al., 2008; Liang et al., 2016; Jia et al., 2017). Here, we investigate the protective role of berbamine analogs in a zebrafish model of 
aminoglycoside-induced hair cell death. The study of structural analogs offers the opportunity to identify structural moieties of berbamine that may be responsible for its protective activity and enable the development of a robust and targeted therapeutic, the long-term goal of this work.

The zebrafish has become an increasingly popular vertebrate model in biomedical research, in part due to their small transparent larvae that are produced in large clutches, providing an excellent system for high-throughput drug screening (Ou et al., 2009, 2010; Coffin et al., 2010; Esterberg et al., 2013; Kenyon et al., 2017). Zebrafish and other fishes possess a sensory system known as the lateral line which is composed of hair cells clustered into groups (neuromasts). These hair cells are structurally and functionally similar to mammalian hair cells and show similar responses to ototoxic damage. The lateral line functions to detect vibrations in the water and to initiate an appropriate behavioral response such as predator avoidance or schooling. One advantage of using this model is that the hair cells are located externally, allowing for easy pharmacological manipulation in vivo. Using this advantage, chemical genetic screens have been performed to identify compounds that protect against aminoglycosideinduced hair cell loss. To date, the zebrafish lateral line has served as a platform for the discovery of several drug candidates with otoprotective potentials, such as PROTO- 1 (the precursor to ORC-13661), berbamine, tacrine, afimoxifene, and linopirdine (e.g., Ton and Parng, 2005; Owens et al., 2008; Ou et al., 2009; Vlasits et al., 2012; Kruger et al., 2016; Kenyon et al., 2017). Furthermore, some of these drugs confer similar protections in the mammalian inner ear (Ou et al., 2009; Kenyon et al., 2017; Kirkwood et al., 2017; Majumder et al., 2017; Chowdhury et al., 2018; Kitcher et al., 2019; O’Reilly et al., 2019).

Here, we report the synthesis and study of 16 berbamine analogs as potential otoprotective therapeutics. We demonstrate that alkylated and acylated berbamine analogs attenuate aminoglycoside-induced hair cell death in the zebrafish lateral line. Tetrahydroisoquinoline monomers were inactive and failed to attenuate cell death. The majority of active analogs confer protection by attenuating aminoglycoside entry into hair cells. Interestingly, a subset of analogs also confer protection via an uptake-independent mechanism. The compounds that show a multimodal mechanism of protection may be stronger leads for developing a therapy because they can potentially modulate multiple targets to inhibit hair cell death. This work demonstrates the potential clinical effectiveness of berbamine analogs as a therapeutic to prevent aminoglycoside ototoxicity.

\section{MATERIALS AND METHODS}

\section{Compound Synthesis}

All solvents and chemicals for compound synthesis were purchased from Acros Organics, Sigma-Aldrich, Combi-Blocks, or AstaTech, Inc. and were used as received. All evaporations were carried out in vacuo with a rotary evaporator. Nuclear magnetic resonance spectra for proton $\left({ }^{1} \mathrm{H}\right.$ NMR) were recorded on a Bruker $300 \mathrm{MHz}$ NMR spectrometer. The chemical shift values are expressed in ppm (parts per million) relative to tetramethylsilane as an internal standard: s, singlet; d, doublet; t, triplet; q, quartet; m, multiplet; br, broad singlet. The relative integrals of peak areas agreed with those expected for the assigned structures. Elemental analyses were performed by AtlanticMicrolab, Incorporation (Norcross, GA, USA). Element compositions are within $0.4 \%$ of the calculated values. Thin-layer chromatography (TLC) was performed on WHATMAN UV254 silica gel plates with a fluorescent indicator and the spots were visualized under 254 and/or $365 \mathrm{~nm}$ illumination. Flash chromatography was performed on a Teledyne ISCO CombiFlash chromatography system using pre-packed silica gel columns purchased from Teledyne ISCO.

\section{Animal Husbandry}

All experiments were approved by the Institutional Animal Care and Use Committee at Washington State University (protocol \# 6024) or by the Animal Care and Use Committee at the National Institutes of Health (NIH; protocol \#1362-13). Zebrafish (Danio rerio) were maintained in aquatic facilities at Washington State University Vancouver or the NIH (calcium imaging experiments only). Zebrafish embryos were obtained by breeding pairs of adults and raising the embryos at $28^{\circ} \mathrm{C}$. Unless stated otherwise, all experiments were conducted using E2 Embryo Medium

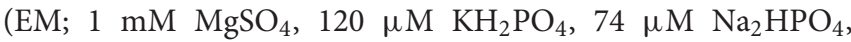
$1 \mathrm{mM} \mathrm{CaCl}_{2}, 500 \mu \mathrm{M} \mathrm{KCl}, 15 \mathrm{mM} \mathrm{NaCl}_{2}$ in distilled water at a $\mathrm{pH}$ of 7.2; Westerfield, 2000). Larval zebrafish between 5-6 days post-fertilization ( $\mathrm{dpf})$ in the wildtype ${ }^{\star} \mathrm{AB}$ strain background were used for experiments unless specifically noted. Tg(myo6b:EGFP), $\mathrm{Tg}($ Brn3c:GFP) or $\mathrm{Tg}$ ( $\alpha$-tubulin: tdTomato) transgenic lines were used to visualize hair cells for a subset of experiments. We selected fish between 5-6 dpf because their hair cells show adult-like responses to ototoxic damage (Murakami et al., 2003; Santos et al., 2006). For calcium imaging experiments to detect mechanosensitive responses in lateral line hair bundles, the previously described transgenic line was used: Tg(-6myo6b:GCaMP6s-CAAX) $)^{i d c 1 T g}$ (Zhang et al., 2018). This line was maintained in the wildtype Tübingen strain background.

\section{Berbamine Analog Dose-Response}

To create a dose-response curve, zebrafish (in 6-well plates) were pretreated with varying log and half-log concentrations $(0.0032 \mu \mathrm{M}$ to $9.98 \mu \mathrm{M})$ of a berbamine analog for one hour before aminoglycoside treatment (neomycin or gentamicin, both from Sigma-Aldrich, St. Louis, MO, USA, N1142 and G1397, respectively). Analogs were reconstituted in dimethylsulfoxide (DMSO) and diluted in EM before zebrafish administration. The dilutions were controlled so the final \% of DMSO was the same across all analogs $(0.5 \%)$. Control fish were treated with DMSO (vehicle) in the absence of analog. Larval zebrafish were exposed to one of two aminoglycoside treatment paradigms, defined here as either "acute" or "chronic" exposure. For acute treatment, zebrafish were exposed to $200 \mu \mathrm{M}$ neomycin for $30 \mathrm{~min}$ in the presence of an analog, followed by four rinses in EM and a 60-min recovery in EM with no drugs present. Chronic exposure consisted of $100 \mu \mathrm{M}$ gentamicin for $6 \mathrm{~h}$ followed by four rinses in EM and a 15-min recovery. We selected these aminoglycosides and exposure paradigms because they both induce hair cell 
damage with distinct time courses and mechanisms (Owens et al., 2009; Coffin et al., 2013a,b).

\section{Hair Cell Assessment}

The majority of our hair cell assessment was done using 2(4-(dimethylamino)styryl)-N- ethylpyridinium iodide (DASPEI; ThermoFisher, Eugene, OR, USA, D426), a mitochondrial dye that robustly labels the hair cells in the lateral line of zebrafish and other fishes (Harris et al., 2003; Coffin et al., 2009; Brown et al., 2010). After aminoglycoside and/or analog treatments, zebrafish were incubated for $15 \mathrm{~min}$ at $28^{\circ} \mathrm{C}$ with $0.005 \%$ DASPEI. The fish were subsequently rinsed $2 \times$ with $\mathrm{EM}$ and anesthetized with $0.001 \%$ buffered tricaine methanesulfonate (MS-222, Syndel, Ferndale, WA, USA) before visualization. We assessed the relative fluorescence intensity for 10 head neuromasts on every zebrafish (IO1, IO2, IO3, IO4, M2, O2, MI1, MI2, SO1, SO2; Raible and Kruse, 2000) using 50x magnification on a Leica M165F fluorescent dissecting microscope. Each neuromast was given a score to quantify the fluorescence: 2 (bright labeling), 1 (modest labeling), and 0 (no labeling); the individual neuromast scores were added to provide an overall score between 0-20 per fish (Harris et al., 2003; Coffin et al., 2009; Owens et al., 2009; Kruger et al., 2016). Scores were averaged per treatment group. To normalize the data, all treatment group averages were divided by the average value of the controls for that experiment and multiplied by 100 to arrive at the percentage of surviving hair cells. DASPEI labeling is a viable method of assessing hair cells with comparable results to directly counting immunolabeled hair cells (Harris et al., 2003; Coffin et al., 2013a; Kruger et al., 2016). For a subset of experiments, we used $\mathrm{Tg}(\mathrm{Brn} 3 \mathrm{c}: \mathrm{GFP})$ larvae, where hair cells express membrane-specific GFP. These experiments provided observation of cellular morphology following aminoglycoside and analog treatment. Animals were treated as described above for analog and aminoglycoside exposure, then euthanized with $0.002 \%$ buffered MS-222, fixed in $4 \%$ paraformaldehyde, and imaged on a Leica SP8 confocal microscope.

\section{Protection After $\mathbf{2 4} \mathbf{~ h}$}

To determine whether we were observing true protection of hair cells from aminoglycoside damage rather than a delay in hair cell death, we exposed zebrafish to the optimal protective concentration (OPC) of each of our analogs for $1 \mathrm{~h}$ followed by a co-treatment with analog and $200 \mu \mathrm{M}$ gentamicin for $30 \mathrm{~min}$, with a 24 -h recovery. This time course was selected to examine the protective capacity of our analogs in a prolonged phase of aminoglycoside exposure since gentamicin-induced hair cell damage continues after drug removal (Owens et al., 2009). Zebrafish were then rinsed $4 \times$ in EM and anesthetized with MS-222 before DASPEI labeling and hair cell assessment.

\section{GTTR Loading Assay}

To quantify the uptake of aminoglycosides in the presence of berbamine analog, we exposed our zebrafish to gentamicin conjugated with Texas Red (GTTR), which predominantly enters hair cells via the MET channel (Steyger et al., 2003; Alharazneh et al., 2011). Zebrafish were first administered a one-hour pretreatment of the optimally protective concentration of analog followed by co-treatment with analog and $50 \mu \mathrm{M}$ GTTR (made according to Steyger et al., 2003) for 18 min (Steyger et al., 2003; Alharazneh et al., 2011; Kruger et al., 2016). The fish were then rinsed $4 \times$ in EM before euthanasia with $0.002 \%$ buffered MS-222 and fixation in 4\% paraformaldehyde, then imaged on a Leica SP8 confocal microscope using a $20 \times$ dry objective with $5 \times$ digital zoom. The gain and laser settings were constant across all groups. The image stacks were collapsed into maximum projection images before image analysis via ImageJ. The images were analyzed by subtracting the background and measuring the fluorescence intensity (arbitrary units; a.u.) of each neuromast and taking an overall average per group (Kruger et al., 2016). The IO2 neuromast was selected for consistency throughout the experiments.

\section{Washout Experiment}

To further understand the mechanism(s) of protection by berbamine analogs, we exposed zebrafish to one of three treatment paradigms that varied in the timing of aminoglycoside administration relative to berbamine analog administration. The first paradigm relied on a pre-co treatment where fish were exposed to the analog both before and during gentamicin exposure. In the second paradigm, there was a co-treatment of the analog and aminoglycoside only during gentamicin exposure. In the third paradigm, post-treatment, the analog was applied after gentamicin removal. In all three treatments, we used a 30-min exposure to $200 \mu \mathrm{M}$ gentamicin followed by a 5.5$\mathrm{h}$ recovery in EM or (for the post-treatment time course) berbamine analog (Kruger et al., 2016). This aminoglycoside time course was selected since gentamicin-induced hair cell damage continues after ototoxin removal, allowing us to assess the optimal protective paradigm in a slower phase of hair cell death (Owens et al., 2009). Zebrafish were then rinsed $4 \times$ in EM and anesthetized with MS-222 before the DASPEI hair cell assessment.

\section{Hair Cell Viability Assessment}

To confirm that our analogs were not compromising the viability of the hair cells or the function of the MET channel after prolonged analog exposure and subsequent removal, we assessed the hair cells using a combination of two vital dyes, YO-PRO1 and FM 1-43FX (both from ThermoFisher Scientific, Eugene, OR, USA, Y3603 and F35355, respectively). YO-PRO-1 is a nucleic acid stain that specifically labels lateral line hair cells in live larvae, allowing us to visualize nuclear morphology (Santos et al., 2006). It is also a nontoxic dye that can be retained for $24 \mathrm{~h}$ making it suitable for long-term hair cell assessments (Thomas et al., 2015; Kruger et al., 2016; Neveux et al., 2016). FM1-43FX is a membrane probe that readily enters hair cells via the MET channel (Gale et al., 2001; Corey et al., 2004; Alharazneh et al., 2011) making it a good proxy for MET channel function following analog removal. Zebrafish were exposed to $3 \mu \mathrm{M}$ YOPRO-1 for $1 \mathrm{~h}$ followed by three rinses in EM. After labeling, the zebrafish were then incubated with analog for $24 \mathrm{~h}$, rinsed $4 \times$ with EM, and left to recover for an hour to allow for dissociation of the analog from the MET channel (e.g., Kirkwood et al., 2017). Zebrafish were then incubated with $1.5 \mu \mathrm{M}$ FM1-43FX 
for $30 \mathrm{~s}$, rinsed $4 \times$ in EM, and anesthetized with MS-222 before live imaging on a Leica SP8 confocal microscope. All imaging was done sequentially on live anesthetized fish to resolve discrete signals between the fluorophores since FM1-43FX has a broad emission spectrum that overlaps with YO-PRO-1. In a separate experiment, $\mathrm{Tg}($ myo6b:EGFP) zebrafish also were used to confirm that berbamine analogs are not ototoxic. We exposed our $\mathrm{Tg}$ (myo6b:EGFP) zebrafish to the OPC of our analogs for $24 \mathrm{~h}$ followed by $4 \times$ rinses in EM. The zebrafish were then euthanized with MS-222 followed by fixation with $4 \%$ paraformaldehyde before confocal imaging of neuromast IO2 for all zebrafish. The gain was kept constant across all groups.

\section{Fluorescent Analog Localization}

We examined a fluorescently tagged berbamine analog to determine if analogs can enter hair cells. We used $\mathrm{Tg}(\alpha$-tubulin: tdTomato) zebrafish (to visualize hair cells) and pre-treated them with a fluorescent 7-(diethylamino)coumarin-tagged berbamine analog (BA-17) for one hour in EM before confocal imaging. The absorption and emission maxima of the coumarin tag are $409 \mathrm{~nm}$ and $473 \mathrm{~nm}$, respectively. To test that the fluorescent tag did not interfere with the protective effects of the analog, we pretreated zebrafish for one hour with the fluorescently tagged analog before acute neomycin exposure. Zebrafish were then rinsed $4 \times$ with EM and anesthetized with MS-222 before DASPEI labeling and hair cell assessment. To examine if the punctate labeling from BA-17 colocalized with lysosomes, we used AB zebrafish labeled with LysoTracker (ThermoFisher, Eugene, OR, USA, L7528). Zebrafish were pretreated with BA-17 for $1 \mathrm{~h}$ followed by a 3-min exposure to $50 \mathrm{nM}$ LysoTracker before confocal imaging of the IO2 neuromast.

\section{Functional Calcium Imaging in Zebrafish Hair Bundles}

The protocol to image GCaMP6s-based calcium signals in zebrafish hair bundles has been described previously in detail (Lukasz and Kindt, 2018). Briefly, before imaging, individual larvae were anesthetized with $0.03 \%$ MS-222 (Sigma) and then pinned onto a Sylgard-filled recording chamber. To suppress the movement, alpha-bungarotoxin $(125 \mu \mathrm{M}$, Tocris, Minneapolis, $\mathrm{MN}$, USA) was injected into the heart. Larvae were then immersed in extracellular imaging solution (in mM: $140 \mathrm{NaCl}$, $2 \mathrm{KCl}, 2 \mathrm{CaCl}_{2}, 1 \mathrm{MgCl}_{2}$, and 10 HEPES, pH 7.3) without MS-222. A fluid-jet (a glass pipette connected to an HSPC-1 device, ALA Scientific, Farmingdale, NY, USA) was used to apply a 500-ms anterior or posterior stimulus to deflect the apical bundles of trunk neuromasts (L1-L4). After baseline GCaMP6s responses were recorded, the extracellular imaging solution was exchanged with the same solution containing a berbamine analog. The solution in the fluid-jet pipette was also exchanged. Ten minutes after the solutions were exchanged, the same hair bundles were restimulated and the GCaMP6s responses were reexamined. Calcium imaging was performed blind concerning the protective capacity of each berbamine analog.

To capture calcium-dependent GCaMP6s signals in apical hair bundles, we used a Bruker Swept-field confocal system equipped with a Rolera EM-C2 CCD camera (QImaging) and a Nikon CFI Fluor $60 \times 1.0$ NA water immersion objective. To coordinate stimulation with image acquisition, the fluid-jet was driven by a voltage-step command from the imaging software (Prairie view) during image acquisition. To simultaneously image the calcium signals in all hair bundles a piezoelectric motor (PICMA P-882.11-888.11 series, PI instruments, Auburn, MA, USA) attached to the objective was used to allow rapid imaging in five planes along the $\mathrm{Z}$-axis at $0.5 \mu \mathrm{m}$ intervals. $\mathrm{Z}$-stacks were acquired at a $50 \mathrm{~Hz}$ frame rate yielding a $10 \mathrm{~Hz}$ volume rate. Calcium imaging experiments were performed on a minimum of four animals and seven neuromasts on two independent days. For image processing and quantification, the five plane Z-stack at each timepoint was projected into a single plane. The GCaMP6s signals in the projected images were then measured in FIJI. In FIJI, a circular region of interest with a $\sim 1.5 \mu \mathrm{m}$ diameter was placed on the center of each bundle and a peak finding program was used to determine the $\Delta \mathrm{F} / \mathrm{F}_{0}$ for each bundle. We quantified both the average bundle $\Delta \mathrm{F} / \mathrm{F}_{0}$ per neuromast and the temporal representation of the average $\Delta \mathrm{F} / \mathrm{F}_{0}$ signals.

\section{Data Analysis}

Data were analyzed via one-way ANOVA using GraphPad Prism version 8. Post hoc comparisons were performed using Bonferroni or Tukey corrections for multiple comparisons (see figure legends for details). For calcium imaging, statistical differences in hair bundle calcium responses were determined using a paired, two-tailed Student's $t$-test. Data are presented as mean \pm SEM. ED $_{50}$ analysis was conducted by normalizing all $y$-values and analyzing dose-response stimulus for agonist concentration vs. normalized response using GraphPad Prism version 8 .

\section{RESULTS}

\section{Berbamine Analog Synthesis}

Berbamine is a bisbenzylisoquinoline comprised of two structurally different benzyltetrahydroisoquinolines in which the benzyl groups are linked on one end and the isoquinoline groups are linked on the other, forming a macrocycle (Figure 1A). We selected an analog synthesis strategy based on the chemistry of the parent berbamine compound, to explore alkyl and phenyl substitutions and the contribution of the dual ring structure to otoprotective activity. Berbamine has one readily available synthetic handle, the phenol, which was used to synthesize our analogs (Figure 1A). This synthetic strategy maintained both the $\mathrm{O}$-acylated (E6 berbamine) and O-alkylated (isotetrandrine) version of berbamine, based on our prior data demonstrating that both of these structures confer otoprotection (Kruger et al., 2016). Eight acylated berbamine analogs were synthesized as shown in Figure 1B (BA-1 to BA-8). Analogs were synthesized using the Steglich esterification method by reacting berbamine with a carboxylic acid in the presence of $\mathrm{N}, \mathrm{N}^{\prime}$-dicyclohexylcarbodiimide and 4-dimethylaminopyridine. Four alkylated berbamine analogs were synthesized (BA-9 to BA-12). These were synthesized using the standard Williamson etherification conditions by reacting berbamine with sodium hydride, and then alkylating the intermediate phenoxide anion 


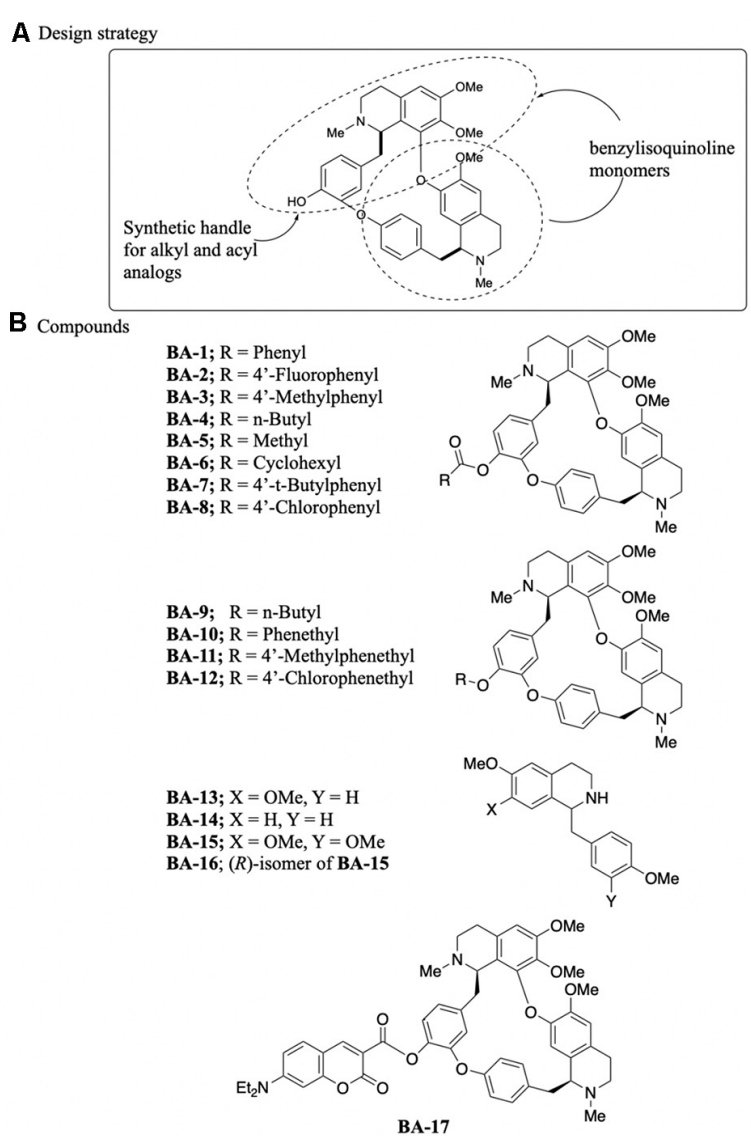

FIGURE 1 | Synthesis of berbamine analogs. (A) Design strategy, including the synthetic handle. (B) Specific analogs synthesized for otoprotection studies. BA-13 through BA-16 are monomers, while BA-17 is a fluorescent analog.

with an alkyl bromide in the presence of a catalytic amount of sodium iodide. These two approaches enable the study of various analogs modified on the phenol position.

Four benzyltetrahydroisoquinolines (BA-13 to B16) were also studied and considered monomers of the bisbenzylisoquinoline ring system. BA-15 and BA-16 were commercially available. BA-13 and BA-14 were synthesized using a slightly modified literature procedure based on Perrey et al. (2013). 4-Methoxyphenylacetic acid was converted into amides with either 4-methoxyphenethylamine or 3, 4-dimethoxyphenethylamine using propanephosphonic acid anhydride as a coupling reagent and Hünig's base. The resulting amides were then cyclized into intermediate dihydroisoquinolines using phosphorous oxychloride in toluene at $90^{\circ} \mathrm{C}$ followed by reduction of the resulting imine with sodium borohydride to form the final compounds.

\section{Berbamine Analogs Confer Dose-Dependent Protection}

We examined these 16 different berbamine analogs and found that all 12 acylated or alkylated analogs (BA-1 to BA-12) protected hair cells from at least one of the aminoglycosides (Figures 2, 3) and were more robust protectants than berbamine. Twelve total analogs significantly protected against acute neomycin damage, while 13 conferred protection from chronic gentamicin. The berbamine monomer benzyltetrahydroisoquinolines conferred little to no protection; BA-14 was significantly protective from gentamicin, and BA-15 was significantly protective from neomycin, but in both cases, protection was modest compared to the other analogs. We observed some toxicity for some analogs for the highest concentration tested $(9.98 \mu \mathrm{M})$ but all protective concentrations were orders of magnitude lower than the ototoxic concentration.

Given our long-term goal of clinical translation, we were especially interested in the analogs with both high efficacy and high potency. As shown in Figure 3, for all 11 acylated or alkylated analogs that attenuated neomycin damage, the OPC (optimal protective concentration) was between $0.032 \mu \mathrm{M}$ and 1 $\mu \mathrm{M}$ with an $\mathrm{ED}_{50}$ range of $0.02 \mu \mathrm{M}-0.24 \mu \mathrm{M}$ (Figures 3A,C). BA-7 (purple line) was the most potent analog with an OPC of $0.032 \mu \mathrm{M}$ and an $\mathrm{ED}_{50}$ of $0.02 \mu \mathrm{M}$. All 12 acylated or alkylated analogs reduced gentamicin toxicity with OPCs varying between $0.32 \mu \mathrm{M}$ and $9.98 \mu \mathrm{M}$ and an $\mathrm{ED}_{50}$ range of 0.03 $\mu \mathrm{M}-1.83 \mu \mathrm{M}$ (Figures 3B,D). Compound BA-8 (pink line) was an exceptionally robust protectant, with an OPC of $0.32 \mu \mathrm{M}$ against gentamicin, yet no protection from neomycin. Overall, the phenyl ring appears to be important, but BA-9, which is an alkylated version of berbamine (and an extension of the alkyl group of isotetrandrine) is also robustly protective, so the phenyl group may not be needed. The statistical analyses for all neomycin and gentamicin dose-response curves can be found in Table 1. Collectively, these data suggest that acylated and alkylated berbamine analogs are important for hair cell protection due to the bisbenzylisoquinoline ring system and the hydrophobic functional groups.

\section{Hair Cells Are Viable and Functional After Prolonged Analog Exposure}

Next, we wanted to assess whether our analogs were compromising the long-term viability of the hair cells or the functionality of the MET channel after prolonged analog exposure and removal. After a 24-h incubation in each analog at the OPC, we assessed hair cell viability using the vital dye YO-PRO-1 and found no qualitative reduction in fluorescence intensity (Figure 4A). FM 1-43FX was used to assess MET channel function since this dye is known to enter hair cells via the MET channel (Gale et al., 2001; Alharazneh et al., 2011). Of the analogs tested that showed protection from neomycin and/or gentamicin, none of them appeared to reduce FM 1-43FX entry, suggesting that MET channel integrity is preserved after analog removal (Figure 4A). To further complement this experiment, berbamine analogs were individually administered for $24 \mathrm{~h}$ to myo6b:EGFP fish to observe hair cell morphology or to $\mathrm{AB}$ larval zebrafish for hair cell survival assessment with DASPEI labeling (Figures 4B,C). We did not observe changes to hair cell morphology or reductions in DASPEI scores. Overall, our viability and functional assays indicate that at their OPC, our analogs do not cause hair cell toxicity. 
A

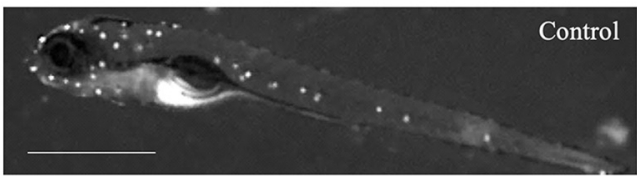

B

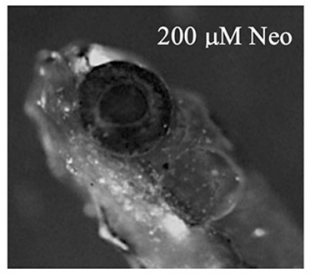

C

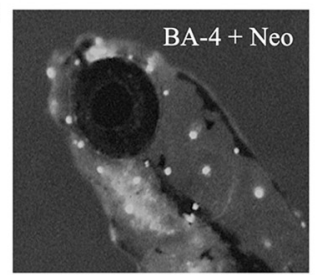

D

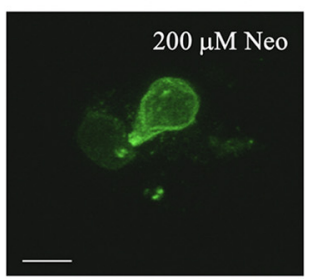

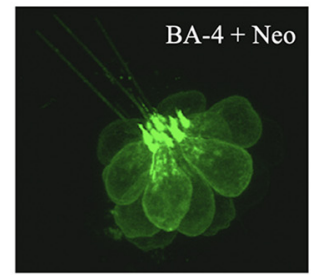

FIGURE 2 | Berbamine analogs confer protection from neomycin. Analog BA-4 is shown here as an example. (A) Zebrafish labeled with the mitochondrial dye DASPEI to visualize neuromasts in the lateral line (white dots). Scale bar $=1 \mathrm{~mm}$. (B) Zebrafish labeled with DASPEl after $200 \mu \mathrm{M}$ neomycin resulting in reduced fluorescence. (C) Zebrafish labeled with DASPEl after pre-co treatment with analog BA-4 and $200 \mu \mathrm{M}$ neomycin. (D,E) Representative IO3 neuromasts from Tg(Brn3c:GFP) zebrafish. (D) Zebrafish exposed to $200 \mu \mathrm{M}$ neomycin exposure, resulting in hair cell loss. Scale bar $=10 \mu \mathrm{m}$. (E) Zebrafish exposed to a pre-co treatment of analog BA-4 and $200 \mu \mathrm{M}$ neomycin.

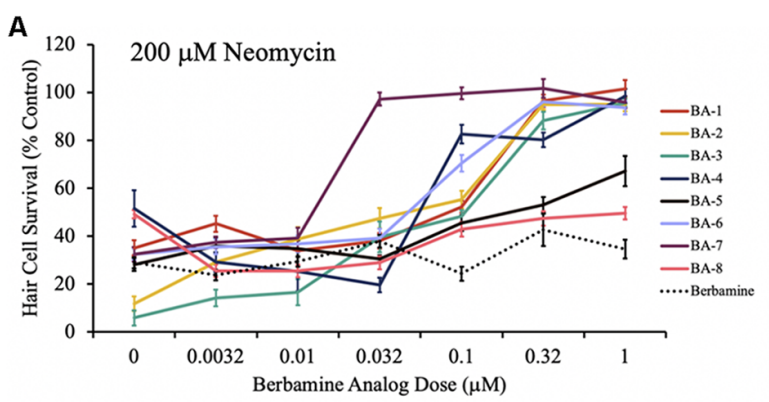

C

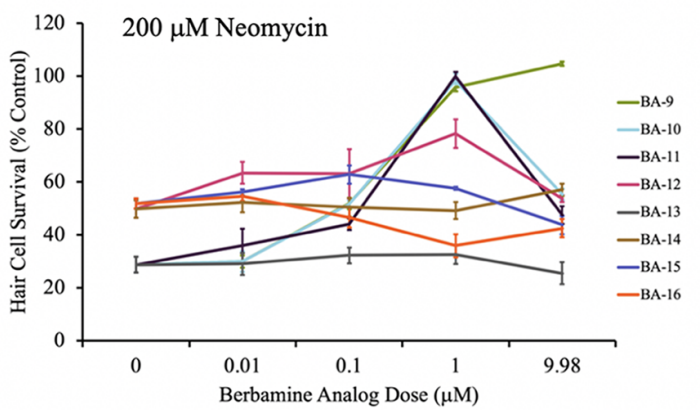

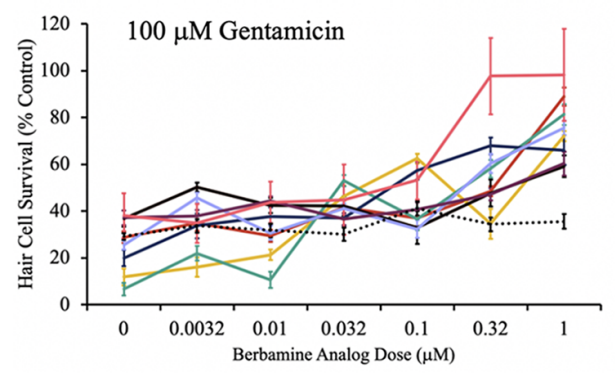

D

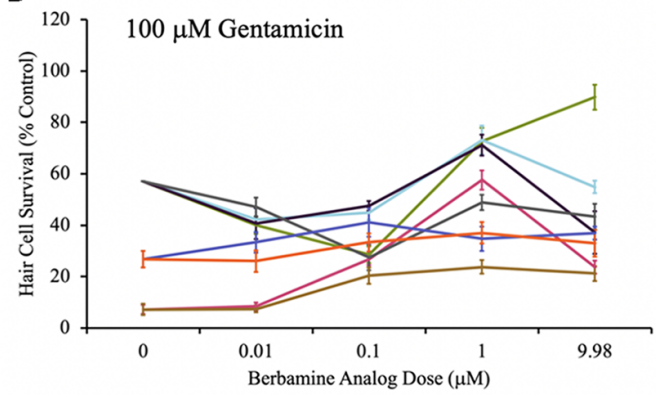

FIGURE 3 | Berbamine analogs confer protection from aminoglycosides. (A,B) Dose-response curves for berbamine analog polymers and berbamine (parent compound) against $200 \mu \mathrm{M}$ neomycin and $100 \mu \mathrm{M}$ gentamicin. (C,D) Dose-response curves for the remainder of the berbamine analog polymers, and the berbamine analog monomers (BA-13 to BA-16) against $200 \mu \mathrm{M}$ neomycin and $100 \mu \mathrm{M}$ gentamicin. Zebrafish were pretreated for $1 \mathrm{~h}$ with each berbamine analog at variable doses followed by co-treatment with analog and aminoglycoside. Data are normalized to controls. Twelve analogs protected hair cells from neomycin damage, while 11 protected from gentamicin. Ten of the berbamine analogs were protective against both neomycin and gentamicin in a dose-dependent manner. Hair cells were assessed via DASPEI. Data are presented normalized to vehicle controls. Data were analyzed by one-way ANOVA, $N=4-12$, except for the gentamicin dose-response curves for BA-9, BA-10, BA-11, and BA-13, where $N=2$ for the gentamicin-only groups. We are still confident in the data for these groups, based on the additional gentamicin data shown here and the data in Supplementary Figure S1. Bars are \pm SEM. Refer to Table 1 for statistics.

Given that our protective analog concentrations are not inherently ototoxic, we next wanted to test whether berbamine analogs were truly otoprotective or if they only delayed hair cell death. We therefore pre-treated larvae with the OPC of analog for one hour before a 30-min administration of gentamicin, then assessed hair cells $24 \mathrm{~h}$ later. This time course was selected 
TABLE 1 | Statistics for berbamine analog dose-response curves for $200 \mu \mathrm{M}$ neomycin and $100 \mu \mathrm{M}$ gentamicin for the data shown in Figure 3 .

\begin{tabular}{|c|c|c|c|c|}
\hline $\begin{array}{l}\text { Compound } \\
\text { Name }\end{array}$ & $\begin{array}{l}\text { Neomycin: } \\
\text { ANOVA }\end{array}$ & $\begin{array}{l}\text { Neomycin: } \\
p \text {-value by } \\
\text { concentration } \\
(\mu \mathrm{M})\end{array}$ & $\begin{array}{l}\text { Gentamicin: } \\
\text { ANOVA }\end{array}$ & $\begin{array}{c}\text { Gentamicin: } \\
p \text {-value by } \\
\text { concentration } \\
(\mu \mathrm{M})\end{array}$ \\
\hline BA-1 & $\begin{array}{l}F_{(6,69)}=94.79 \\
P<0.0001\end{array}$ & $\begin{array}{c}0.1 ;{ }^{* *} \\
0.32 ;{ }^{* * * *} \\
1 ;{ }^{* * * *}\end{array}$ & $\begin{array}{l}F_{(6,70)}=48.91 \\
P<0.0001\end{array}$ & $\begin{array}{c}0.032 ;{ }^{*} \\
0.32 ;{ }^{* * * *} \\
1 ;{ }^{* * * *}\end{array}$ \\
\hline BA-2 & $\begin{array}{l}F_{(6,66)}=72.23 \\
P<0.0001\end{array}$ & $\begin{array}{c}0.0032 ;{ }^{* *} \\
0.01 ;{ }^{* * * *} \\
0.032 ;{ }^{* * * *} \\
0.1 ;{ }^{* * * *} \\
0.32 ;{ }^{* * * *} \\
1 ;{ }^{* * * *}\end{array}$ & $\begin{array}{l}F_{(8,89)}=50.76 \\
P<0.0001\end{array}$ & $\begin{array}{l}0.032 ;{ }^{* * * *} \\
0.1 ;^{* * * *} \\
0.32 ;{ }^{* * *} \\
1 ;{ }^{* * * *} \\
3.16 ;{ }^{* * * *} \\
9.98 ; * * * *\end{array}$ \\
\hline BA-3 & $\begin{array}{l}F_{(6,70)}=70.76 \\
P<0.0001\end{array}$ & $\begin{array}{l}0.032 ;{ }^{* * *} \\
0.1 ;^{* * * *} \\
0.32 ;{ }^{* * * *} \\
1 ; ;^{* * * *}\end{array}$ & $\begin{array}{l}F_{(8,63)}=61.08 \\
P<0.0001\end{array}$ & $\begin{array}{c}0.0032 ;^{*} \\
0.032 ;{ }^{* * * *} \\
0.1 ;^{* * * *} \\
0.32 ;^{* * * * *} \\
1 ;^{* * * *} \\
3.16 ;{ }^{* * * *} \\
9.98 ; ;^{* * * *}\end{array}$ \\
\hline BA-4 & $\begin{array}{l}F_{(6,70)}=51.23 \\
P<0.0001\end{array}$ & $\begin{array}{c}0.0032 ;{ }^{* *} \\
0.01 ;{ }^{* *} \\
0.032^{* * * *} \\
0.1 ;{ }^{* * * *} \\
0.32 ;{ }^{* * * *} \\
1 ;{ }^{* * * * *}\end{array}$ & $\begin{array}{l}F_{(6,60)}=19.36 \\
P<0.0001\end{array}$ & $\begin{array}{c}0.01^{*} \\
0.032 ;{ }^{*} \\
0.1 ;{ }^{* * * *} \\
0.32 ;{ }^{* * * * *} \\
1 ;{ }^{* * * *}\end{array}$ \\
\hline BA-5 & $\begin{array}{l}F_{(6,68)}=15.29 \\
P<0.0001\end{array}$ & $\begin{array}{c}0.1 ;^{* *} \\
0.32 ;^{* * * *} \\
1 ;{ }^{* * * *}\end{array}$ & $\begin{array}{c}F_{(6,42)}=4.34 \\
P=0.017\end{array}$ & $\begin{array}{c}0.0032 ;{ }^{*} \\
1 ;{ }^{* * *}\end{array}$ \\
\hline BA-6 & $\begin{array}{l}F_{(6,70)}=94.12 \\
P<0.0001\end{array}$ & $\begin{array}{c}0.1 ;^{* * * * *} \\
0.32 ;{ }^{* * * * *} \\
1 ;{ }^{* * * *}\end{array}$ & $\begin{array}{l}F_{(6,76)}=41.35 \\
P<0.0001\end{array}$ & $\begin{array}{c}0.0032 ;{ }^{* * * * *} \\
0.032 ;{ }^{* * *} \\
0.32 ;^{* * * *} \\
1 ;{ }^{* * * *}\end{array}$ \\
\hline BA-7 & $\begin{array}{l}F_{(6,53)}=113.8 \\
P<0.0001\end{array}$ & $\begin{array}{c}0.032 ; * * * * \\
0.1 ;^{* * * *} \\
0.32 ; ;^{* * * *} \\
1 ;{ }^{* * * *}\end{array}$ & $\begin{array}{l}F_{(8,77)}=10.83 \\
P<0.0001\end{array}$ & $\begin{array}{l}1 ;{ }^{* * *} \\
3.16 ; \\
\text { **** }\end{array}$ \\
\hline BA-8 & $\begin{array}{l}F_{(6,72)}=19.58 \\
P<0.0001\end{array}$ & $\begin{array}{l}0.0032 ;{ }^{* * * * *} \\
0.01 ;{ }^{* * * * *} \\
0.032 ;{ }^{* * * *}\end{array}$ & $\begin{array}{l}F_{(8,59)}=19.85 \\
P<0.0001\end{array}$ & $\begin{array}{c}0.32 ; * * * * \\
1 ; * * * * \\
3.16 ; * * * *\end{array}$ \\
\hline BA-9 & $\begin{array}{l}F_{(4,40)}=332.3 \\
P<0.0001\end{array}$ & $\begin{array}{l}0.1 ; * * * * \\
1 ; * * * * \\
9.98 ; * * * *\end{array}$ & $\begin{array}{l}F_{(4,38)}=32.05 \\
P<0.0001\end{array}$ & $\begin{array}{l}0.1 ;{ }^{*} \\
9.98 ;{ }^{*}\end{array}$ \\
\hline BA-10 & $\begin{array}{l}F_{(4,39)}=111.5 \\
P<0.0001\end{array}$ & $\begin{array}{l}0.1 ;{ }^{* * * *} \\
1 ; * * * * \\
9.98 ; * * * *\end{array}$ & $\begin{array}{l}F_{(4,33)}=9.09 \\
P<0.0001\end{array}$ & ns \\
\hline BA-11 & $\begin{array}{l}F_{(4,26)}=87.80 \\
P<0.0001\end{array}$ & $\begin{array}{l}0.1 ;{ }^{* *} \\
1 ;{ }^{* * * *} \\
9.98 ;\end{array}$ & $\begin{array}{l}F_{(4,23)}=7.09 \\
P=0.0007\end{array}$ & ns \\
\hline BA-12 & $\begin{array}{l}F_{(4,37)}=5.90 \\
P=0.0009\end{array}$ & $1 ;{ }^{* * *}$ & $\begin{array}{l}F_{(4,52)}=67.35 \\
P<0.0001\end{array}$ & $\begin{array}{l}0.1 ; \text { ***** }^{*} \\
1 ;{ }^{* * * *} \\
9.98^{* * * *}\end{array}$ \\
\hline BA-13 & $F_{(4,32)}=0.60$ & Ns & $\begin{array}{c}F_{(4,22)}=5.18 \\
P=0.004\end{array}$ & $0.1 ;{ }^{\star \star}$ \\
\hline $\mathrm{BA}-14$ & $F_{(4,44)}=0.468$ & Ns & $\begin{array}{l}F_{(4,48)}=10.54 \\
P<0.0001\end{array}$ & $\begin{array}{l}0.1 ;{ }^{* * *} \\
1 ;{ }^{* * * *} \\
9.98 ; * * *\end{array}$ \\
\hline BA-15 & $\begin{array}{l}F_{(4,48)}=8.03 \\
P<0.0001\end{array}$ & $0.1 ; *$ & $\begin{array}{c}F_{(5,34)}=1.80 \\
P=0.139\end{array}$ & ns \\
\hline $\mathrm{BA}-16$ & $\begin{array}{l}F_{(4,40)}=5.99 \\
P=0.0007\end{array}$ & $1 ;{ }^{* *}$ & $\begin{array}{c}F_{(5,34)}=1.29 \\
P=0.289\end{array}$ & ns \\
\hline
\end{tabular}

TABLE 1 | Continued

\begin{tabular}{llccc}
\hline $\begin{array}{l}\text { Compound Neomycin: } \\
\text { Name }\end{array}$ & ANOVA & $\begin{array}{c}\text { Neomycin: } \\
\boldsymbol{p} \text {-value by } \\
\text { concentration } \\
(\boldsymbol{\mu} \mathbf{M})\end{array}$ & $\begin{array}{c}\text { Gentamicin: } \\
\text { ANOVA }\end{array}$ & $\begin{array}{c}\text { Gentamicin: } \\
\boldsymbol{p} \text {-value by } \\
\text { concentration } \\
(\boldsymbol{\mu} \mathbf{M})\end{array}$ \\
\hline Berbamine & $\begin{array}{l}F_{(6,60)}=4.02 \\
P=0.0019\end{array}$ & $0.32^{*}$ & $\begin{array}{c}F_{(6,66)}=2.14 \\
P=0.06\end{array}$ & $\mathrm{~ns}$ \\
& & &
\end{tabular}

Compound names in bold indicate protection from both neomycin and gentamicin, while compound names in black (not bold) protect from neomycin or gentamicin, and compound names in gray indicate non-protective compounds. Data were analyzed by one-way ANOVA (columns 2 and 4) followed by Bonferroni-corrected $t$-tests for multiple comparisons (columns 3 and 5). In columns 3 and 5, numbers in black indicate significant increases in hair cell survival (compared to aminoglycoside-only controls), while numbers in gray indicate significant reductions in hair cell survival relative to aminoglycoside-only groups. ${ }^{* * * *} p<0.0001,{ }^{* * *} p<0.001,{ }^{* *} p<0.01,{ }^{*} p<0.05$. ns, not significant.

because after an acute gentamicin exposure hair cell damage continues even after drug removal (Owens et al., 2009). All of the analogs tested (BA-2, BA-6, BA-7, and BA-8) showed over 90\% hair cell survival $24 \mathrm{~h}$ post-gentamicin exposure compared to untreated controls (Figure 5). When we compared the protection of our analogs in chronic $(6 \mathrm{~h})$ vs. acute $(30 \mathrm{~min}+24 \mathrm{~h})$ gentamicin treatment, we saw that BA- 6 and BA-7 exhibited $10-20 \%$ more protection in the acute gentamicin exposure assay (compare Figures 3B, 5). These data demonstrate that berbamine analogs robustly protect hair cells from aminoglycoside damage after both acute and chronic aminoglycoside treatment.

\section{A Subset of Berbamine Analogs Reduce GTTR Loading}

Next, we investigated if our analogs protect hair cells by blocking aminoglycoside entry. Previous research shows that aminoglycosides predominantly enter hair cells via the MET channel. Furthermore, quinoline-ringed structures have been shown to protect hair cells by reducing MET channel-mediated aminoglycoside loading (Ou et al., 2012; Kirkwood et al., 2017). Since berbamine is a quinoline ring structure we predicted its analogs could also protect by reducing aminoglycoside uptake via the MET channel. We found that all 12 of the acylated or alkylated berbamine analogs (BA-1 to BA-12) attenuated GTTR uptake by $50 \%$ or more relative to negative controls (Figures 6A,B). The berbamine monomers (BA-13 to BA-16) did not attenuate uptake compared to negative controls. Calcium, which reduces the open probability of the MET channel, was used as a positive control. While calcium reduced GTTR by $70 \%, 10$ of our analogs were more effective uptake blockers, as was the parent compound berbamine (Figure 6B, Kruger et al., 2016). BA-7 was the most effective uptake blocker with over 95\% attenuation of GTTR fluorescence (Figure 6B). We then conducted a linear regression to determine how strongly hair cell protection was correlated with GTTR uptake (Figure 6C). Given a correlation coefficient of 0.23 , there is no relationship, suggesting that protection may not be solely reliant on aminoglycoside uptake block. Overall our GTTR uptake analysis indicates that a subset of analogs block GTTR loading into the hair cells but the magnitude of uptake block is not directly correlated with hair cell protection. 
A
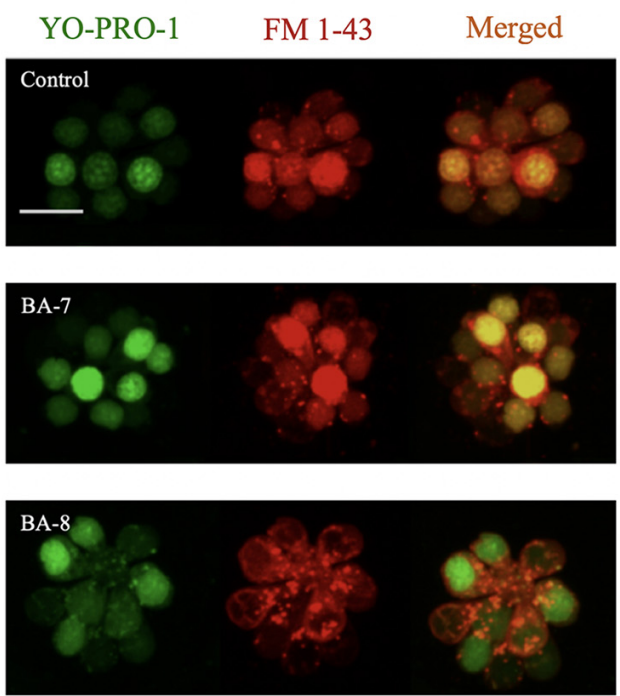

B
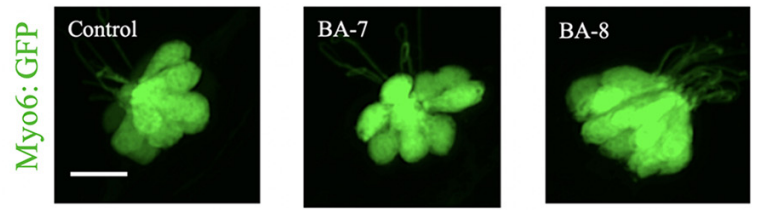

C

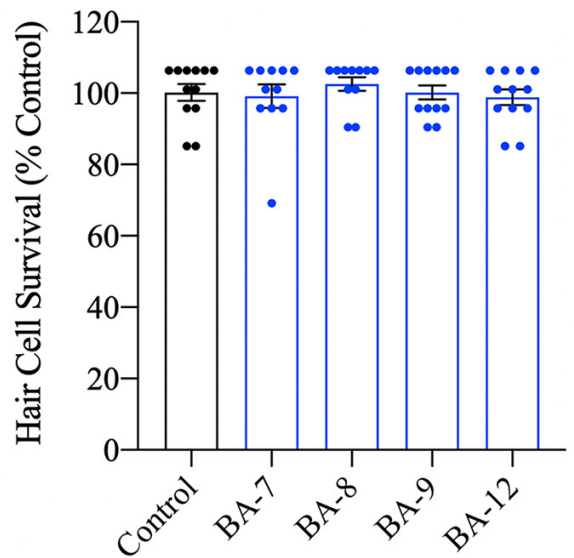

FIGURE 4 | Hair cells are viable after analog exposure. (A) Zebrafish were pretreated with the nuclear marker YO-PRO-1 for $1 \mathrm{~h}$ followed by treatment with the optimally protective concentration of each analog for $24 \mathrm{~h}$. Zebrafish were then rinsed in embryo medium (EM) and treated with FM 1-43FX to assess mechanoelectrical transduction (MET) channel function after prolonged analog exposure and removal. Analog treatment did not alter FM 1-43FX or YO-PRO-1-labeling. Scale bar $=10 \mu \mathrm{m}$. (B) Examples of Tg(myo6b:EGFP) neuromasts treated with the optimally protective concentration of each analog for $24 \mathrm{~h}$ before hair cell assessment. In each case, neuromast morphology appeared normal. Scale bar $=10 \mu \mathrm{m}$. (C) Zebrafish were exposed to the optimal protective concentration (OPC) of berbamine analog for $24 \mathrm{~h}$ before hair cell assessment with DASPEI. None of the analogs impact hair cell survival. Data are normalized to controls and analyzed by one-way ANOVA, $F_{(4,48)}=0.6564, p=0.6252$. $N=11-12$, bars are \pm SEM.

\section{Berbamine Analogs Differentially Affect Mechanoelectrical Transduction Channel Activity}

Our results indicate that several of our otoprotective berbamine analogs attenuate aminoglycoside toxicity by blocking GTTR uptake through MET channels. To confirm that protective analogs impair mechanotransduction, we mechanically stimulated lateral line hair cells and measured GCaMP6s-evoked calcium signals in hair bundles (Lukasz and Kindt, 2018). This previously established method allows for sensitive and quantitative measurements of mechanotransduction in lateral line hair bundles (Figure 7A). Therefore, these GCaMP6s signals can provide a quantitative readout of mechanosensitive-calcium responses in the presence and absence of berbamine analogs. For our analysis, we tested two representative analogs, one protective analog (BA-9, a potent GTTR blocker) and one analog that was not protective (BA-16, which does not block GTTR uptake). We first measured evoked GCaMP6s signals in hair bundles during fluid-jet stimulation before analog treatment (control, blue line, Figures 7B-E). After the application of the berbamine monomer BA-16, there was no difference in the magnitude of the GCaMP6s signals in hair bundles as compared to the pretreatment condition, indicating that BA-16 does not impair mechanotransduction (Figures 7D,E).
By contrast, the application of the berbamine derivative BA-9 reduced the GCaMP6s signals by $\sim 95 \%$, indicating significant inhibition of mechanotransduction (Figures $7 \mathrm{~B}, \mathrm{C}$ ). Our calcium imaging experiments complement the GTTR uptake data and suggest that when berbamine analogs block GTTR uptake, their otoprotective effects are due to the MET channel block, thereby reducing aminoglycoside entry into hair cells.

\section{Berbamine Analogs May Also Protect via Uptake-Independent Mechanisms}

We have shown that many of our berbamine analogs can confer protection when co-administered with gentamicin. Given that 12 of our berbamine analogs are likely to be otoprotective due to reduced GTTR uptake, we hypothesized that no protection would be conferred if we administered the analogs after gentamicin removal. To test this hypothesis, we used an acute 30-min gentamicin treatment time course. We compared three gentamicin treatment paradigms: (1) 1$\mathrm{h}$ pre-treatment with the analog, followed by co-treatment with analog and gentamicin (pre-co); (2) co-treatment with the analog-only during gentamicin treatment (no pre-treatment; co) and (3) analog post-treatment for $5.5 \mathrm{~h}$ after gentamicin washout (post). All experiments used the OPC of each analog (Figure 8). 


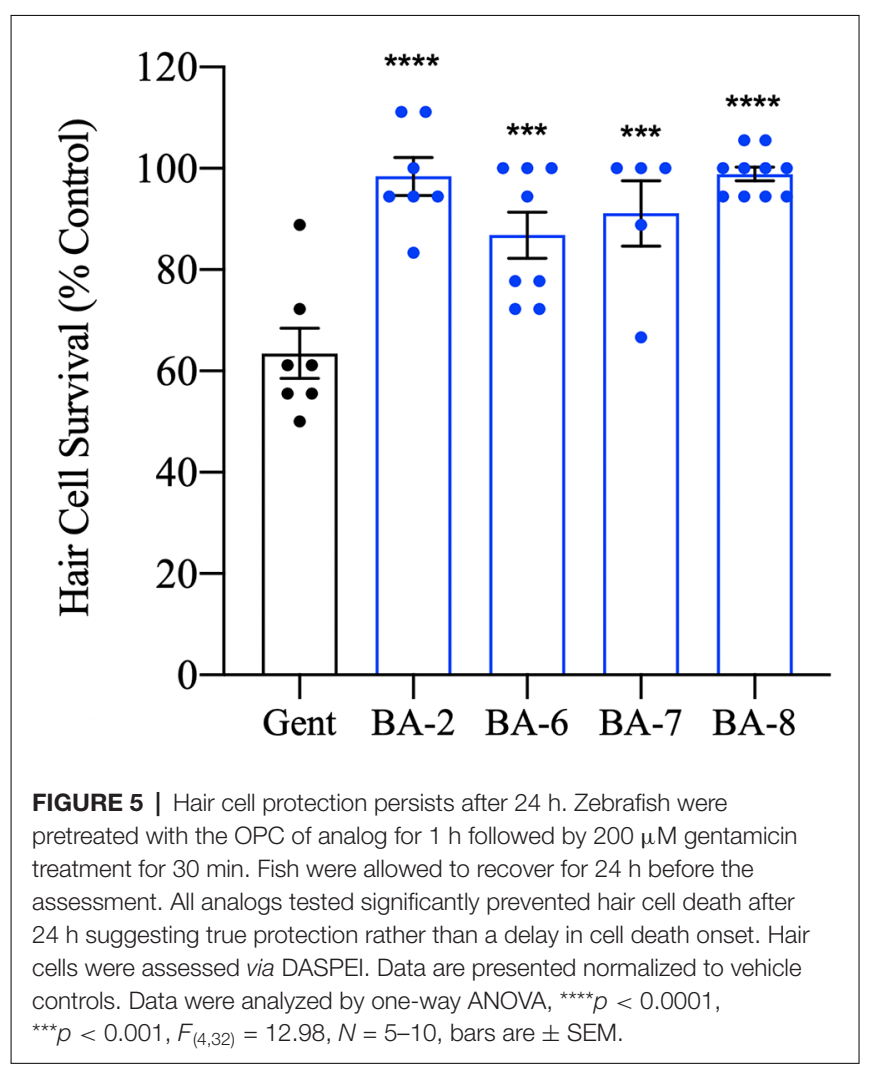

Consistent with our hypothesis, eight of our analogs (BA-1, BA-2, BA-4, BA-8, BA-9, BA-10, BA-11, and BA-12) only protected hair cells when the analog was administered before and during (pre-co) or concurrent with (co) aminoglycoside administration (Figures 8A-C, Supplementary Figure S1, Supplementary Table S1). One analog, BA-5, which was previously not protective when co-administered with gentamicin in the chronic assay, was protective when administered before and during gentamicin exposure (preco). This suggests that protection occurs via aminoglycoside uptake block in an acute gentamicin administration but that protection diminishes in a longer treatment paradigm. Surprisingly, BA-3, BA-6, and BA-7 showed protection in all three treatment paradigms. Analogs that show protection even when administered after aminoglycoside removal suggest that hair cell protection may also occur by an uptake-independent mechanism such as interaction with intracellular pathways.

\section{Berbamine Analogs Enter Hair Cells}

Given that a subset of analogs protects hair cells by both uptake-dependent and uptake-independent mechanisms, we next wanted to know if the analogs enter hair cells. For these experiments, we used the approach outlined in Figure 1 to synthesize BA-17, a berbamine analog with a fluorescent 7-(diethylamino)coumarin tag. To assess for protection by this fluorescent analog, zebrafish were exposed to varying concentrations of analog before and concurrently with aminoglycoside exposure. We found that BA-17 was protective in a dose-dependent manner against $200 \mu \mathrm{M}$ neomycin and toxic at a concentration of $9.98 \mu \mathrm{M}$, consistent with other protective analogs (Figure 9A). To determine whether our analogs enter hair cells, we pre-treated $T g(\alpha-t u b u l i n: t d T o m a t o)$ zebrafish with the OPC of BA-17 for one hour before confocal imaging. BA-17 was visible in hair cells as well as some punctae in surrounding neuromasts, suggesting the analog can enter multiple cell types (Figure 9B). To begin to understand cellular localization of berbamine analogs, we asked if our analogs enter lysosomes. For these experiments, we pre-treated AB zebrafish for $1 \mathrm{~h}$ with BA-17 followed by incubation in LysoTracker and found that some BA-17 punctae colocalize with the lysosomal marker (Figure 9C). These data demonstrate that berbamine analogs can enter neuromasts cells and localize in subcellular compartments, including lysosomes.

\section{DISCUSSION}

Our former work demonstrated that berbamine, a natural plant alkaloid, robustly protected zebrafish hair cells from aminoglycoside-induced damage (Kruger et al., 2016). In a separate study, Kirkwood et al. (2017) further confirmed berbamine's otoprotective properties in the zebrafish lateral line and in mouse cochlear explants. The present study set out to develop berbamine analogs that are more potent and efficacious than the parent compound and to investigate the extent of their protection against aminoglycosideinduced hair cell damage. The medicinal chemistry campaign strategy focused on functionalizing the phenol group to synthesize analogs as an initial probe of the structure-activity relationship. Leveraging available chemistry in this manner is a robust strategy employed in the early development of natural products. Like many natural products, the berbamine scaffold is somewhat complex making de novo synthesis far more time consuming and expensive than substitution at the available phenol. E6 berbamine and isotetrandrine, which also confer protection from aminoglycosides (Kruger et al., 2016), are both substituted at the phenol suggesting further exploration at that position would likely produce active compounds. Moreover, since MET inhibition was the likely target of activity, increasing analog size and hydrophobicity by substitution at the phenol should make analogs better-MET blockers.

Overall, we show that many of our berbamine analogs effectively protect hair cells from aminoglycoside toxicity, demonstrating more robust protection than berbamine. Details of all 16 non-fluorescent analogs are shown in Figure 10, where we classify analogs based on the relative degree of protection. Of the 16 analogs, nine demonstrated at least $40 \%$ protection against both neomycin and gentamicin-induced hair cell death ("robust" or "strong" protection, see Figure 10). Two analogs protected against neomycin (>30\% protection) but conferred little protection from gentamicin (BA-5, BA12) and one only conferred protection from gentamicin but not neomycin (BA-8). Twelve analogs reduced GTTR and thus likely confer protection by blocking aminoglycoside entry through the MET channel, which is the primary site 
A
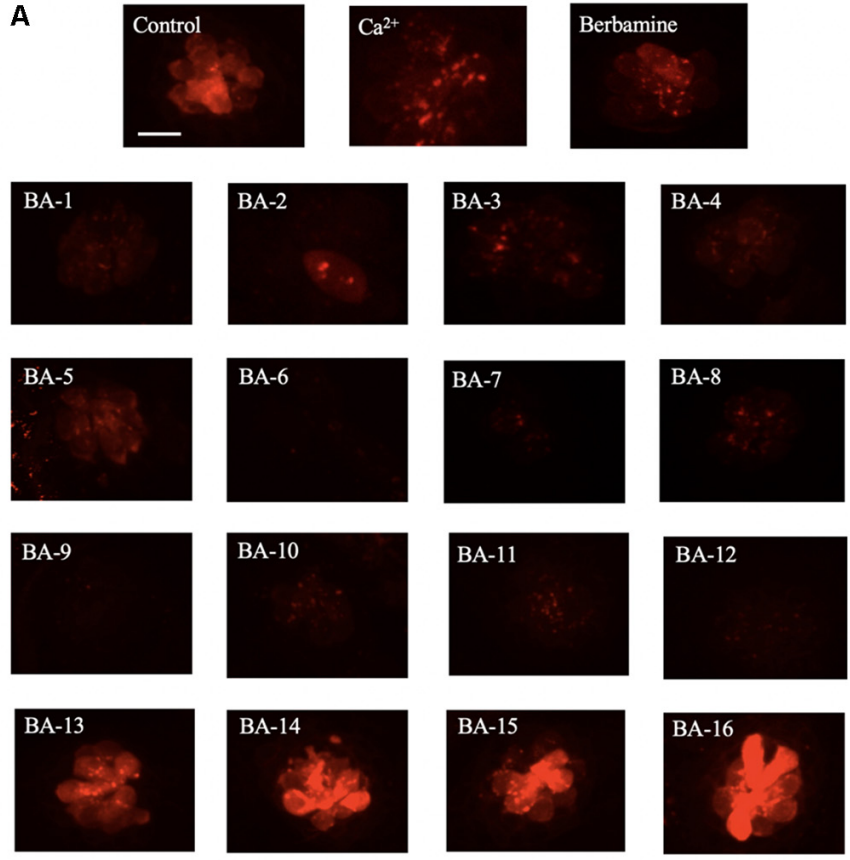

B

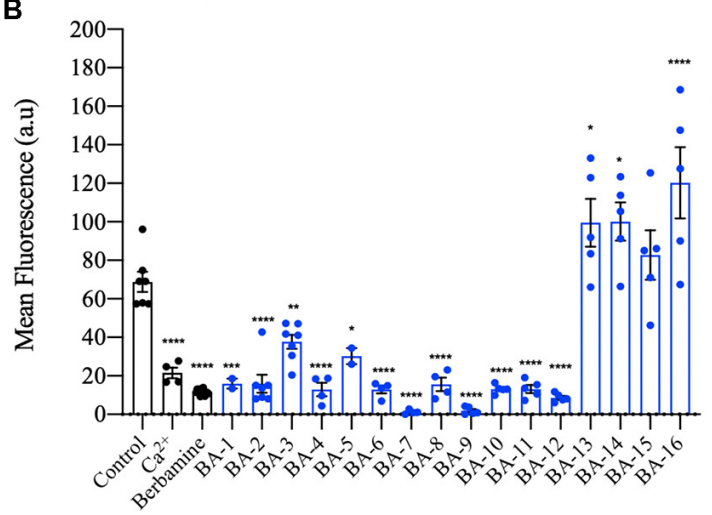

C

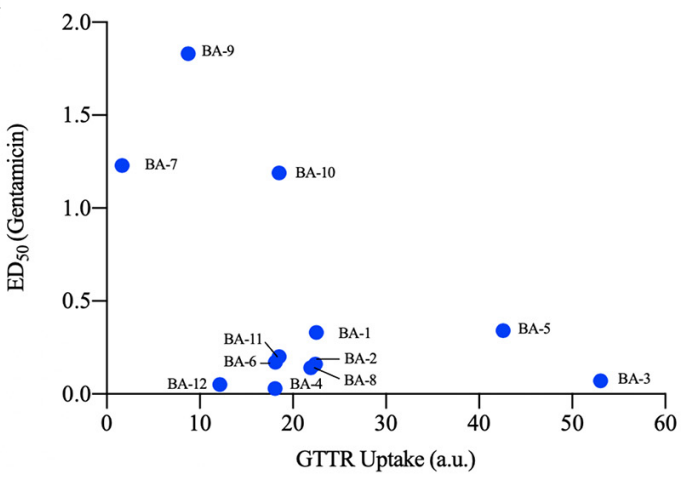

FIGURE 6 | Berbamine analogs reduce aminoglycoside uptake by hair cells. (A) Twelve of the analogs significantly reduced uptake of gentamicin conjugated with Texas Red (GTTR), as did berbamine. Zebrafish were pretreated with the optimally protective analog concentration (or with $25 \mu \mathrm{M}$ berbamine, Kruger et al., 2016) for $1 \mathrm{~h}$ followed by a co-treatment with $50 \mu \mathrm{M}$ GTTR for $18 \mathrm{~min}$. Dimethylsulfoxide (DMSO) and high calcium are negative and positive controls, respectively. (B) Fluorescence intensity quantification using the OPC of analog. The intensity was measured in arbitrary units and the data were normalized by subtracting the background fluorescence. Data were analyzed via one-way ANOVA, $F_{(17,101)}=38.71,{ }^{* \star *} p<0.0001,{ }^{* \star *} p<0.001,{ }^{* \star} p<0.01,{ }^{*} p<0.05$, asterisks denote significantly different from vehicle controls, $N=4-8$ for most compounds, except for $N=2$ for compounds BA- 1 and BA-5 and $N=15$ for berbamine, bars are \pm SEM. (C) Linear regression was conducted for analogs that protect hair cells from gentamicin to identify a relationship between analog potency and uptake $(y=-0.0203 x+0.9162)$. The Pearson correlation coefficient of 0.2268 denotes no significant relationship. Berbamine data are replotted from Kruger et al. (2016).

of aminoglycoside entry into hair cells (Marcotti et al., 2005; Alharazneh et al., 2011). BA-9, a potent uptake blocker and protective compound against both neomycin and gentamicin, reduced MET-evoked calcium activity to baseline levels, further supporting our hypothesis that protection is largely due to MET channel block. The reduction of MET-evoked calcium activity was not observed with BA16 , a non-protective compound. Our results are consistent with Kirkwood et al. (2017), who demonstrated that berbamine is a permeant MET channel blocker in mouse cochlear hair cells.

Attenuating aminoglycoside uptake into hair cells has gained traction as a feasible otoprotective strategy (Ou et al., 2012; O’Sullivan et al., 2017; Kitcher et al., 2019). d-Tubocurarine and phenoxybenzamine have previously been shown to protect hair cells by reducing aminoglycoside entry (Kirkwood et al., 2017; Majumder et al., 2017). d-Tubocurarine is a structural analog of berbamine with a dimethyl quaternary ammonium 


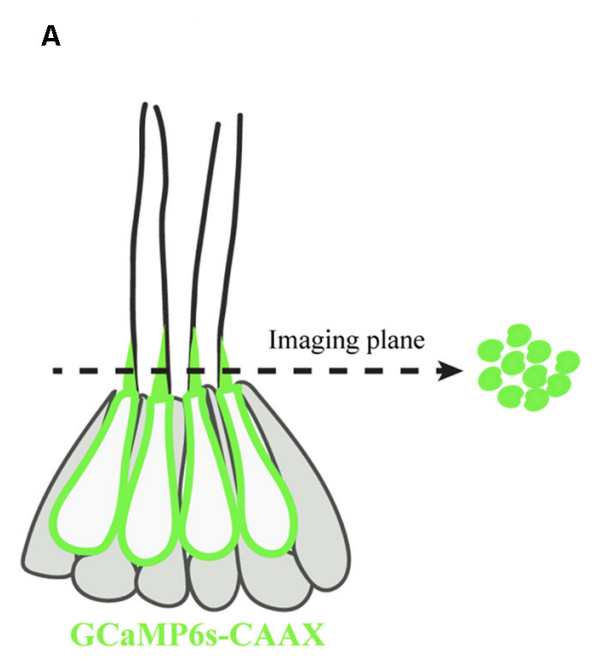

B

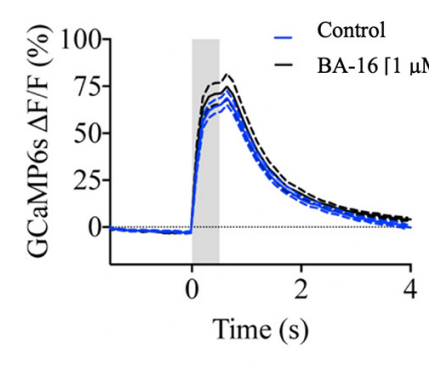

D

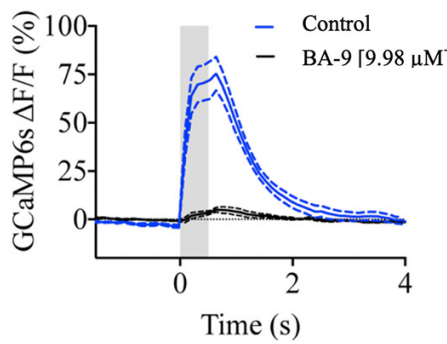

C

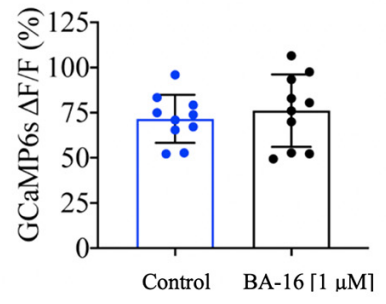

E

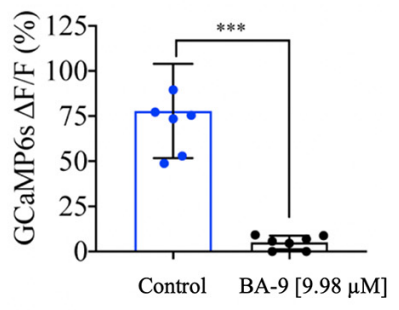

FIGURE 7 | Berbamine analogs differentially reduce mechanotransduction channel activity. Mechanosensitive-calcium signals from hair bundles of posterior lateral line neuromasts. (A) Side view of a neuromast expressing hair cell-specific membrane-localized GCaMP6s. Left: an imaging plane through the apical hair bundles can be used to measure mechanosensitive-calcium signals during fluid-jet stimulation. Right: a top-down view of the imaging plane showing the hair bundles expressing GCaMP6s. (B) Traces comparing the average GCaMP6s signals before and after application of $1 \mu \mathrm{M}$ BA-16, $N=10$ neuromasts. (C) Dot plots quantifying the peak GCaMP6s response per neuromast. BA-16 does not alter evoked calcium activity in hair cells. (D) Traces comparing the average GCaMP6s signals before and after application of $1 \mu \mathrm{M}$ BA-9, $N=7$ neuromasts. (E) Dot plots quantifying the peak GCaMP6s response per neuromast. BA-9 reduces MET-evoked calcium activity compared to baseline levels. Each dot in $\mathbf{( C , E )}$ averages the response of all hair bundles in each neuromast. A paired $t$-test was used in $(\mathbf{C}, \mathbf{E}),{ }^{* * *} p<0.001$. Error bars in $(\mathbf{C}, \mathbf{E})$ and dashed lines on either side of the solid line in (B,D) represent \pm SEM.

ion in one of the tetrahydroisoquinoline rings and with one of the other methoxy groups replaced with a second phenol (Supplementary Figure S2). Ionic compounds such as d-tubocurarine might be expected to block the MET channel since similar effects were observed with analogs of other scaffolds (Supplementary Figure S2). ORC-13661 is being developed as an otoprotectant and also protects both zebrafish and rodent hair cells from aminoglycosides, likely by blocking aminoglycoside uptake (Chowdhury et al., 2018). Quaternary analogs of ORC-13661 were much more active than the non-quaternary versions. ORC-13661 as well as our berbamine analogs contain lipophilic groups that appear to improve protective activity. Protection is likely due to the hydrophobic nature of the compounds and their ability to diffuse through plasma membranes. This attribute makes these compounds better candidates for future drug development since they could potentially cross the blood-brain barrier and/or the blood labyrinth barrier (Abbott et al., 2006; Koo et al., 2015; Nyberg et al., 2019).

The majority of our protective analogs were not only lipophilic but also contained an aryl group. Absence of the aryl moiety reduced our analogs' overall protection from aminoglycosides, especially from gentamicin, as we found for compound BA-5. Loss of an aryl substituent and its impact on protective activity from aminoglycosides in zebrafish was also observed in Chowdhury et al. (2018). In that study, the original screening hit contained a $p$-chlorophenyl group and replacement led to a loss of activity. In our study, analog BA-8 also contained a chlorophenyl substituent but it did not seem to be important for neomycin protection, in contrast to the Chowdhury et al. (2018) study. However, the chlorophenyl substituent was important for robust gentamicin protection. Likely, differences seen in neomycin protection for the $\mathrm{p}$-chlorophenyl compounds (ORC13661 and BA-8) may have more to do with how the substituent interacts in combination with its compound scaffold to permeate the MET channel rather than channel blocking activity being solely reliant on the moiety.

Nevertheless, aryl groups are present in other protective compounds such as tacrine, berbamine, carvedilol, ORC-13661, and phenoxybenzamine, suggesting the importance of the aryl moiety for otoprotection (Ou et al., 2012; Kruger et al., 2016; Chowdhury et al., 2018; O'Reilly et al., 2019). It is likely that the phenyl group, among other aryl groups, may be necessary for protection due to their unusual stability and ability to delocalize charges within their structure by resonance. This would allow for temporary charges to reside within the structure which may form favorable electrostatic interactions with its target and could explain why quaternary versions of protective drugs such as ORC-13661 are more active. This is further supported by evidence from Chowdhury et al. (2018) and O'Reilly et al. (2019). Both studies found that aminoglycoside otoprotection required a basic center with a positive charge to engage in electrostatic interactions, such as basic nitrogen or a quaternary ammonium salt. An electronegative atom could also yield a temporary dipole and thus a transient charge, which may explain why analogs such as BA-2 and BA-8 were robust 


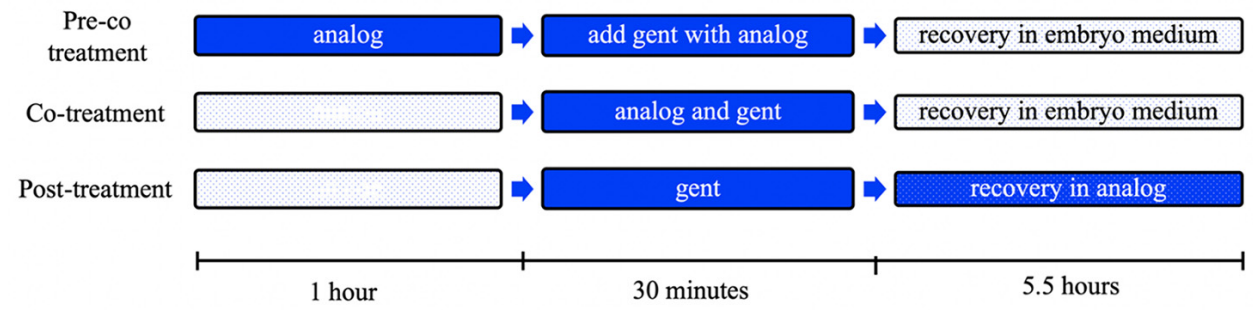

A

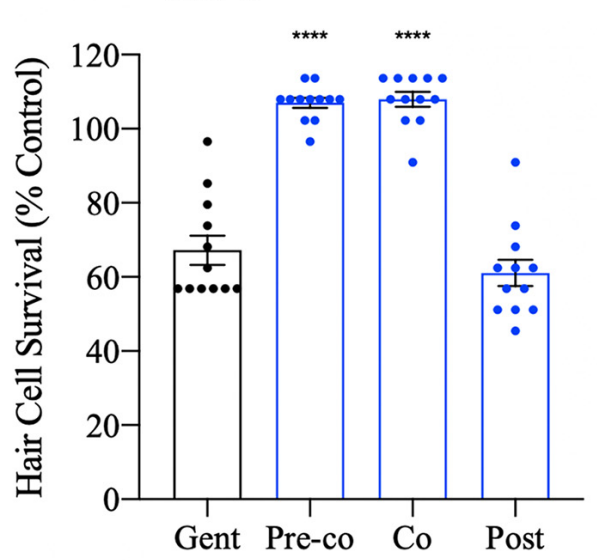

C

\section{BA-7}

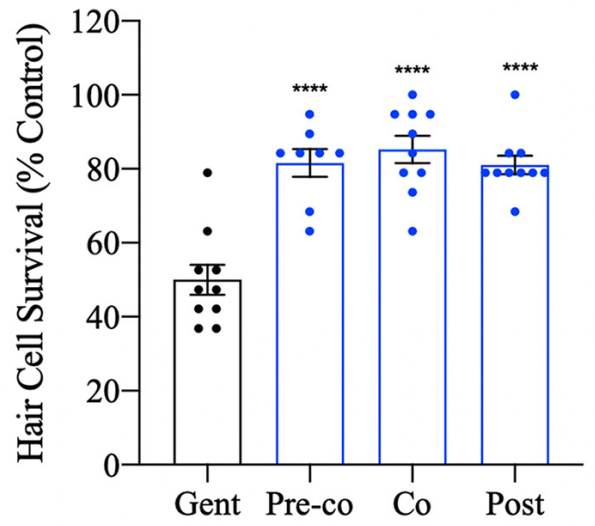

B

BA-6

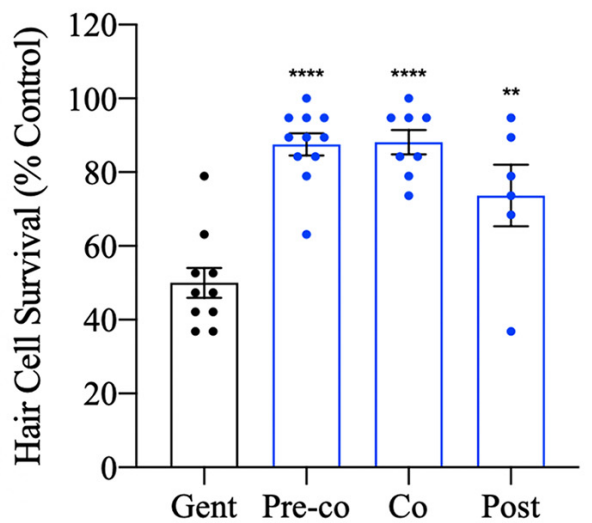

BA-16

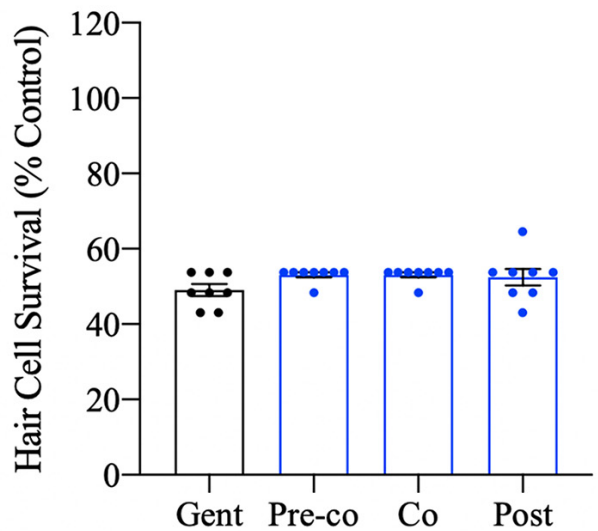

FIGURE 8 | Differential patterns of analog protection from gentamicin. Zebrafish were either pre-treated with the OPC of analog for 1 h, then co-treated with analog and gentamicin for 30 min ("pre-co" condition), co-treated with analog and gentamicin without a pretreatment period ("co" condition) or post-treated with analog for $5.5 \mathrm{~h}$ following gentamicin removal ("post" condition). All treatments used $200 \mu \mathrm{M}$ gentamicin. Examples are shown for analogs that represent each pattern of protection. (A) Analog BA-1 was only protective when administered in the "pre-co" or "co" conditions suggesting that protection occurs via aminoglycoside uptake block. (B,C) Analogs BA-6 and BA-7 significantly protected hair cells in all three conditions, suggesting an intracellular mechanism of protection in addition to aminoglycoside uptake block. (D) Analog BA-16 is not protective of any treatment paradigm. Hair cells were assessed via DASPEI. Data are presented normalized to untreated controls, which are not shown but represent $100 \%$ hair cell survival. Data were analyzed via one-way ANOVA, ${ }^{\star \star * \star} p<0.0001,{ }^{* \star} p<0.01$ (as compared to gentamicin-only controls), $N=7-12$, bars are + SEM. Refer to Supplementary Table S1 for statistics.

MET blockers. However, aromaticity is not the only feature that contributes to otoprotection. The majority of our aromatic compounds were not protective, such as BA-13 through BA-16.
These compounds were half the weight of the other analogs tested. Additionally, analogs BA-13 through BA-16 have a carbon center, similar to some non-protective carvedilol derivatives that 


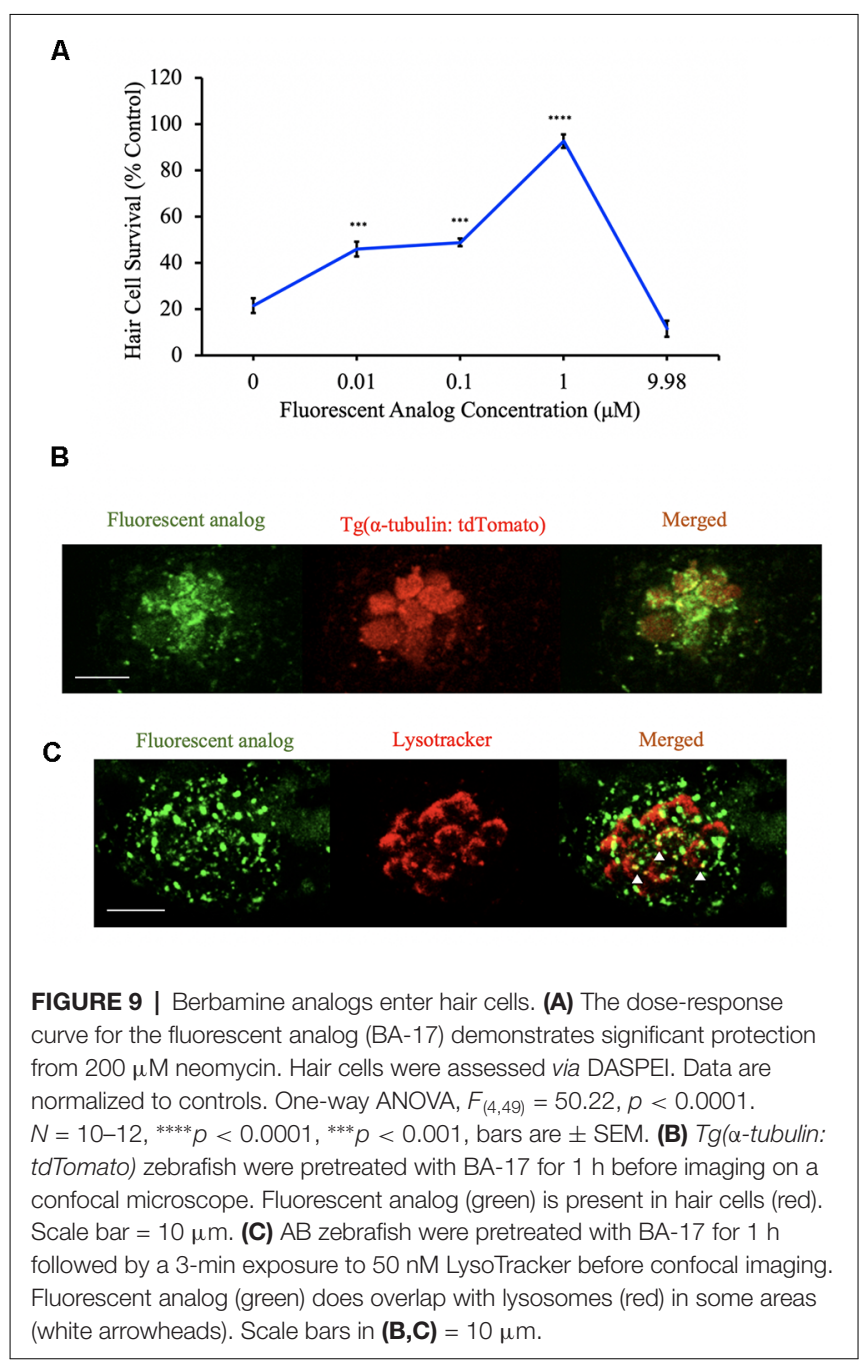

substituted the center nitrogen with carbon or nonbasic nitrogen (O’Reilly et al., 2019).

BA-8 robustly protects hair cells from gentamicin damage yet confers no protection from neomycin. Also, the majority of our protective analogs show differing degrees of protection from neomycin vs. gentamicin, supporting the literature that neomycin and gentamicin activate partially distinct pathways of hair cell damage (Owens et al., 2009; Coffin et al., 2013a,b; Wiedenhoft et al., 2017). BA-5 was only protective when administered before and during gentamicin exposure (pre-co), suggesting protection occurs via transient aminoglycoside uptake block during acute gentamicin administration. In the chronic gentamicin assay, BA-5 was not protective against gentamicin. This could mean that BA-5 may disassociate from the MET channel in a longer treatment paradigm thus rendering it non-protective. These data are consistent with prior studies showing that acute neomycin and acute gentamicin activate similar damage mechanisms, while chronic gentamicin also operates via additional pathway(s) (Coffin et al., 2009, 2013a,b; Owens et al., 2009). Additionally, there is more recent evidence that aminoglycosides enter hair cells via both endocytic and non-endocytic pathways, resulting in drug delivery from the extracellular space to lysosomes, and that intracellular trafficking differs between aminoglycosides (Hailey et al., 2017). Some of our analogs likely target cellular pathways that are unique to neomycin vs. gentamicin, thus accounting for the observed differences in protection.

We observed that the fluorescently modified analog BA-17 does enter hair cells but whether the entry is necessary for protection remains to be determined. An interesting observation from this experiment was the punctate/granular labeling of BA-17 in hair cells, which may co-localize with lysosomes (Figure 9C), supporting findings from Hailey et al. (2017) that both aminoglycosides that enter hair cells ultimately end up in lysosomes in zebrafish. Future experiments will further examine the analogs' mechanism of protection by observing how long the fluorescently tagged analog stays in hair cells and how that time scale compares to the protection that it exhibits. We also found that there is no correlation between the degree of uptake block and the magnitude of hair cell protection. Interestingly, some of the analogs that weren't protective (BA13, BA-16) seemed to enhance GTTR entry. This information could be potentially useful as it suggests that certain moieties may exacerbate toxicity and should be avoided in future drug optimization. Additionally, our analog washout experiments suggest that our analogs disassociate from the channel and do not alter MET channel function after the analog is removed, suggesting that hair cell viability is preserved.

While the MET channel is the primary site of aminoglycoside entry into hair cells, several other ion channels are also candidates for aminoglycoside entry. Transient receptor potential (TRP) channels such as TRPV1, TRPV4, and TRPA1 are particularly interesting because they have pore diameters large enough to allow passage of bulky aminoglycoside molecules (Myrdal and Steyger, 2005; Karasawa et al., 2008; Stepanyan et al., 2011). Kenyon et al. (2017) discovered a select group of aromatic compounds that protect against aminoglycoside-induced hearing loss and are also known to have biological activity for potassium channels, NMDA and AMPA receptors, and other channels. Of the 13 protective analogs identified in their study, only six were MET channel blockers, yet additional compounds reduced fluorescent drug entry, implying that the other compounds may exert their protective effects through other receptors or channels (Kenyon et al., 2017). Bisbenzylisoquinoline compounds, in addition to inhibiting prostaglandins and leukotrienes (Teh et al., 1990) are also proficient vasodilators and modulators of voltagegated calcium channels (Medeiros et al., 2011; Alaoui et al., 2015), leading to the hypothesis that our analogs may reduce aminoglycoside entry through alternate channels in tandem with the MET channel, which would explain the varying degrees of GTTR fluorescence attenuation shown by our analogs. This would also explain why some of our multimodal protective analogs such as BA-3 and BA- 6 are not the strongest of uptake blockers, unlike analogs such as BA-9 and BA-12, which only protect by attenuating aminoglycoside entry. Future studies will further characterize the binding targets of our berbamine analogs, which can also provide insight into the mechanisms of aminoglycoside-induced hair cell death. 


\begin{tabular}{|c|c|c|c|c|c|c|c|c|}
\hline $\begin{array}{l}\text { Compound } \\
\text { Name }\end{array}$ & Structure (R-group) & $\begin{array}{c}\text { MW } \\
(\mathrm{g} / \mathrm{mol})\end{array}$ & $\begin{array}{l}\text { General } \\
\text { Protection } \\
\text { Class }\end{array}$ & $\begin{array}{c}\text { Neo } \\
\text { Protective; } \\
\text { ED } 50(\mu \mathrm{M})\end{array}$ & $\begin{array}{c}\text { Gent } \\
\text { Protective; } \\
\mathrm{ED}_{50}(\mu \mathrm{M})\end{array}$ & $\begin{array}{c}\text { Likely } \\
\text { Mechanism of } \\
\text { Protection }\end{array}$ & $\begin{array}{c}\text { Neo Optimal } \\
\text { Protective } \\
\text { Concentration } \\
(\mu \mathrm{M})\end{array}$ & $\begin{array}{l}\text { Gent Optimal } \\
\text { Protective } \\
\text { Concentration } \\
(\mu \mathrm{M})\end{array}$ \\
\hline BA-1 & Phenyl & 785.7 & Robust & Yes; 0.14 & Yes; 0.33 & MET Blocker & 0.32 & 1 \\
\hline BA-2 & 4'-Fluorophenyl & 803.7 & Robust & Yes; 0.04 & Yes; 0.16 & MET Blocker & 0.32 & 3.16 \\
\hline BA-3 & 4'-Methylphenyl & 815.8 & Strong & Yes; 0.07 & Yes; 0.07 & $\begin{array}{l}\text { MET Blocker } \\
\text { and Uptake- } \\
\text { Independent }\end{array}$ & 1 & 1 \\
\hline BA-4 & n'-Butyl & 765.8 & Strong & Yes; 0.24 & Yes; 0.03 & MET Blocker & 1 & 0.32 \\
\hline BA-5 & Methyl & 723.7 & Modest & Yes; 0.14 & No & MET Blocker & 1 & -- \\
\hline BA-6 & Cyclohexyl & 791.8 & Strong & Yes; 0.08 & Yes; 0.17 & $\begin{array}{l}\text { MET Blocker } \\
\text { and Uptake- } \\
\text { Independent }\end{array}$ & 0.32 & 1 \\
\hline $\mathrm{BA}-7$ & 4'-t- Butylphenyl & 841.9 & Robust & Yes; 0.02 & Yes; 1.23 & $\begin{array}{l}\text { MET Blocker } \\
\text { and Uptake- } \\
\text { Independent }\end{array}$ & 0.032 & 3.16 \\
\hline BA-8 & 4'-Chlorophenyl & 820.2 & Robust & No & Yes; 0.14 & MET Blocker & -- & 0.32 \\
\hline BA-9 & n'-Butyl & 664.84 & Robust & Yes; 0.20 & Yes; 1.83 & MET Blocker & 1 & 9.98 \\
\hline BA-10 & Phenethyl & 712.89 & Robust & Yes; 0.18 & Yes; 1.19 & MET Blocker & 1 & 1 \\
\hline BA-11 & 4'-Methylphenethyl & 726.91 & Robust & Yes; 0.24 & Yes; 0.20 & MET Blocker & 1 & 1 \\
\hline BA-12 & 4'-Chlorophenethyl & 747.33 & Modest & Yes; 0.06 & Yes; 0.05 & MET Blocker & 1 & 1 \\
\hline BA-13 & $\mathrm{OMe}, \mathrm{H}$ & 349.86 & $\begin{array}{c}\text { Not } \\
\text { Protective }\end{array}$ & No & No & No protection & -- & -- \\
\hline BA-14 & $\mathrm{H}$ & 319.83 & $\begin{array}{c}\text { Not } \\
\text { Protective }\end{array}$ & No & No & No protection & -- & -- \\
\hline BA-15 & $\mathrm{OMe}$ & 379.88 & $\begin{array}{c}\text { Not } \\
\text { Protective }\end{array}$ & No & No & No protection & -- & -- \\
\hline BA-16 & $\mathrm{OMe}$ & 379.88 & $\begin{array}{c}\text { Not } \\
\text { Protective }\end{array}$ & No & No & No protection & -- & -- \\
\hline
\end{tabular}

FIGURE 10 | Summary of berbamine analog protection and compound characteristics. Compound name, structure, molecular weight, protection class, likely mechanism of protection, $\mathrm{ED}_{50}$, and OPCs against neomycin and gentamicin for all 16 berbamine analogs. The general protection classes were defined based on thresholds of protection levels by percent. Robust $=60 \%$ increase in hair cell protection compared to aminoglycoside group; Strong $=$ over $40 \%$ increase in hair cell protection compared to aminoglycoside group; Modest = over 30\% increase in hair cell protection compared to aminoglycoside group; No protection = less than $30 \%$ of hair cell protection compared to aminoglycoside group. The likely mechanism was determined by the GTTR experiments in Figure $\mathbf{6}$ and the time course experiments shown in Figure $\mathbf{8}$ and Supplementary Figure $\mathbf{S 1 .}$

Based on our findings that the majority of our analogs protect by blocking aminoglycoside uptake, we expected to only observe protection when the berbamine analog was administered either before or at the same time as the antibiotic. We were surprised that three analogs (BA-3, BA-6, and BA-7) exhibited protection when delivered after aminoglycoside exposure but before hair cell death, suggesting both uptake-dependent and uptake-independent protective mechanisms. Interestingly, all three analogs were alkylated with bulky lipophilic groups such as alkyl-substituted phenyl groups and cyclohexyl analog. Analogs with halide substitution or small straight-chain alkyl substitution did not have the same profile. Berbamine has been used as an anti-inflammatory in traditional Chinese medicine and has gained traction as an anti-cancer agent $(\mathrm{Du}$ et al., 2018). Previous studies have shown that berbamine and its analogs can inhibit tumor growth by modulating nuclear factor kappa-light-chain-enhancer of activated B cells (NF-KB) signaling (Liang et al., 2016; Jia et al., 2017). A recent study showed that berbamine has anti-inflammatory activity that robustly inhibits the release of chemokines and cytokines including TNF- $\alpha$, IL-1 $\beta$, IL-6 (Jia et al., 2017). Berbamine also suppressed the phosphorylation of $\mathrm{p} 65$, І $\kappa \mathrm{B}$, c-Jun N-terminal kinase (JNK) pathways, and extracellular receptor kinase (ERK)1/2 pathways (Jia et al., 2017), all of which are implicated in aminoglycoside-induced hair cell loss (Huth et al., 2011). Possibly the berbamine analogs that confer uptakeindependent protection may reduce pro-inflammatory cytokines or modulate pathways such as NF-KB, JNK, or ERK1/2. Future work will examine the berbamine modulation of intracellular pathways in aminoglycoside-damaged hair cells.

In our prior work, $25 \mu \mathrm{M}$ berbamine was the optimal concentration that prevented aminoglycoside toxicity and 
reduced GTTR loading (Kruger et al., 2016 and Figure 6A). One advantage of our analogs compared to berbamine is that the analogs are protective at a much lower concentration than the parent compound (over 100-fold more potent, in some cases), making the analogs suitable for clinical translation. One caveat of some of our analogs is that they are toxic to hair cells at high concentrations $(9.98 \mu \mathrm{M})$ in zebrafish (Figures 3C,D). This toxicity was also observed when berbamine was tested in mice at concentrations greater than or equal to $30 \mu \mathrm{M}$ (Kirkwood et al., 2017). However, analog toxicity was observed at much higher concentrations than the optimally protective concentration. For example, the OPC for BA-7 is 300 -fold lower than the ototoxic concentration. Toxicity of berbamine and some of its analogs at higher concentrations may be due to the para-methylene phenol moiety that is common to many bisbenzylisoquinolines, which can lead to reactive intermediates (Tian and Zheng, 2017). Furthermore, many clinical drugs such as Amphetamine and Dapsone are toxic at high concentrations but highly effective at low concentrations (Schulz and Schmoldt, 2003). An advantage of using analogs with natural scaffolds is they can be readily modified to enhance desired characteristics such as physiological stability, solubility, and efficiency (Ji et al., 2009). Moving forward we plan to determine the pharmacokinetics of our lead analogs and further investigate the protective mechanism(s) of these leads in rodents as a prelude to clinical trials.

In summary, our study demonstrates that berbamine analogs with phenolic moieties robustly protect against aminoglycosideinduced hair cell death, primarily by attenuating aminoglycoside entry into hair cells, with some analogs also conferring uptakeindependent protection. Several of the berbamine analogs described here were more potent than ORC-13661, a compound in clinical trials as an otoprotectant. The multiple modes of protection of some of our analogs also bolster their clinical use since there are likely multiple relevant targets, meaning that a single compound may confer protection via multiple mechanisms to increase protective efficacy.

Our former work confirmed that berbamine does not interfere with the antibacterial efficacy of aminoglycosides (Kruger et al., 2016) suggesting that berbamine analogs are not likely to interfere with clinical aminoglycoside use but that hypothesis remains to be tested. The zebrafish lateral line is an excellent pre-clinical tool for drug discovery and lead compound optimization for further translational work; future research will address the extent of analog protection in mammals. From our zebrafish analog screen, we identified several analogs, particularly BA-3, BA-4, and BA-7, as leads for future studies; these potent compounds $\left(\mathrm{ED}_{50}<0.25 \mu \mathrm{M}\right.$

\section{REFERENCES}

Abbott, N. J., Rönnbäck, L., and Hansson, E. (2006). Astrocyte-endothelial interactions at the blood-brain barrier. Nat. Rev. Neurosci. 7, 41-53. doi: 10.1038/nrn1824

Alaoui, C. E., Choukairi, Z., Lamri, L., Berrada, F., and Fechtali, T. (2015). Inhibition of voltage-gated calcium channels by natural alkaloids: pharmacological and therapeutic effects. Int. J. Sci. Res. 4, 1289-1294. for neomycin) demonstrate protection from neomycin and gentamicin and strong GTTR uptake block (Figure 10). Our research provides more insight into hair cell pathology and aminoglycoside-induced hearing loss, yielding new approaches to preserve hearing.

\section{DATA AVAILABILITY STATEMENT}

The raw data supporting the conclusions of this article will be made available by the authors, without undue reservation.

\section{ETHICS STATEMENT}

The animal study was reviewed and approved by Institutional Animal Care and Use Committee, Washington State University.

\section{AUTHOR CONTRIBUTIONS}

$\mathrm{AH}$ and $\mathrm{AC}$ conceived the concept, designed the bulk of the experiments and wrote the bulk of the manuscript. ON, JW and BB developed the analog synthesis strategy and made the analogs. KK designed, conducted, and analyzed the calcium imaging experiments. AH, GL and AC conducted all other experiments and analyzed the associated data. All authors contributed to writing and editing.

\section{FUNDING}

This research was funded by the National Institutes of Health Award R15DC013900 and by funds from Washington State University, both to AC. This work was also supported by National Institute on Deafness and Other Communication Disorders Intramural Research Program Grant 1ZIADC00008501 to KK.

\section{ACKNOWLEDGMENTS}

We thank R. Boney for assistance with early experiments, E. Cooper, M. Sokolova, R. Tsay, and M. Macpherson for fish husbandry, K. Lawton for assistance with the calcium imaging experiments, and P.S. Steyger for insightful discussions. We also thank two anonymous reviewers for helpful comments.

\section{SUPPLEMENTARY MATERIAL}

The Supplementary Material for this article can be found online at: https://www.frontiersin.org/articles/10.3389/fncel.2020.002 34/full\#supplementary-material.

Alharazneh, A., Luk, L., Huth, M., Monfared, A., Steyger, P. S., Cheng, A. G., et al. (2011). Functional hair cell mechanotransducer channels are required for aminoglycoside ototoxicity. PLoS One 6:e22347. doi: 10.1371/journal.pone. 0022347

Bainbridge, K. E., and Wallhagen, M. I. (2014). Hearing loss in an aging american population: extent, impact, and management. Annu. Rev. Public Health 35, 139-152. doi: 10.1146/annurev-publhealth-032013182510 
Brown, A. D., Mussen, T. D., Sisneros, J. A., and Coffin, A. B. (2010). Reevaluating the use of aminoglycoside antibiotics in behavioral studies of the lateral line. Hear. Res. 272, 1-4. doi: 10.1016/j.heares.2010.10.014

Chowdhury, S., Owens, K. N., Herr, R. J., Jiang, Q., Chen, X., Johnson, G., et al. (2018). Phenotypic optimization of urea-thiophene carboxamides to yield potent, well tolerated and orally active protective agents against aminoglycoside-induced hearing loss. J. Med. Chem. 61, 84-97. doi: 10.1021/acs.jmedchem.7b00932

Coffin, A. B., Ou, H., Owens, K. N., Santos, F., Simon, J. A., Rubel, E. W., et al. (2010). Chemical screening for hair cell loss and protection in the zebrafish lateral line. Zebrafish 7, 3-11. doi: 10.1089/zeb.2009.0639

Coffin, A. B., Reinhart, K. E., Owens, K. N., Raible, D. W., and Rubel, E. W. (2009). Extracellular divalent cations modulate aminoglycoside-induced hair cell death in the zebrafish lateral line. Hear. Res. 253, 42-51. doi: 10.1016/j.heares.2009. 03.004

Coffin, A. B., Rubel, E. W., and Raible, D. W. (2013a). Bax, Bcl2, and P53 differentially regulate neomycin- and gentamicin-induced hair cell death in the zebrafish lateral line. J. Assoc. Res. Otolaryngol. 14, 645-659. doi: 10.1007/s10162-013-0404-1

Coffin, A. B., Williamson, K. L., Mamiya, A., Raible, D. W., and Rubel, E. W. (2013b). Profiling drug-induced cell death pathways in the zebrafish lateral line. Apoptosis 18, 393-408. doi: 10.1007/s10495-013-0816-8

Corey, D. P., García-Añoveros, J., Holt, J. R., Kwan, K. Y., Lin, S.Y., Vollrath, M. A., et al. (2004). TRPA1 is a candidate for the mechanosensitive transduction channel of vertebrate hair cells. Nature 432, 723-730. doi: 10.1038/nature03066

Du, G.-H., Song, X.-Y., Kong, L.-L., and Chen, N.-H. (2018). Berbamine," in Natural Small Molecule Drugs from Plants, ed. G. Du (Singapore: Springer), 485-489.

Esterberg, R., Coffin, A. B., Ou, H., Simon, J. A., Raible, D. W., and Rubel, E. W. (2013). Fish in a dish: drug discovery for hearing habilitation. Drug Discov. Today Dis. Models 10:10.1016/j.ddmod.2012.02.001. doi: 10.1016/j.ddmod. 2012.02.001

Farris, H. E., LeBlanc, C. L., Goswami, J., and Ricci, A. J. (2004). Probing the pore of the auditory hair cell mechanotransducer channel in turtle. J. Physiol. 558, 769-792. doi: 10.1113/jphysiol.2004.061267

Gale, J. E., Marcotti, W., Kennedy, H. J., Kros, C. J., and Richardson, G. P. (2001). FM1-43 dye behaves as a permeant blocker of the hair-cell mechanotransducer channel. J. Neurosci. 21, 7013-7025. doi: 10.1523/JNEUROSCI.21-18-07 013.2001

Hailey, D. W., Esterberg, R., Linbo, T. H., Rubel, E. W., and Raible, D. W. (2017). Fluorescent aminoglycosides reveal intracellular trafficking routes in mechanosensory hair cells. J. Clin. Invest. 127, 472-486. doi: 10.1172/jci85052

Harris, J. A., Cheng, A. G., Cunningham, L. L., MacDonald, G., Raible, D. W., and Rubel, E. W. (2003). Neomycin-induced hair cell death and rapid regeneration in the lateral line of zebrafish (danio rerio). J. Assoc. Res. Otolaryngol. 4, 219-234. doi: 10.1007/s10162-002-3022-x

Huth, M. E., Ricci, A. J., and Cheng, A. G. (2011). Mechanisms of aminoglycoside ototoxicity and targets of hair cell protection. Int. J. Otolaryngol. 2011:937861. doi: $10.1155 / 2011 / 937861$

Ji, H.-F., Li, X.-J., and Zhang, H.-Y. (2009). Natural products and drug discovery. Can thousands of years of ancient medical knowledge lead us to new and powerful drug combinations in the fight against cancer and dementia? EMBO Rep. 10, 194-200. doi: 10.1038/embor.2009.12

Jia, X.-J., Li, X., Wang, F., Liu, H.-Q., Zhang, D.-J., and Chen, Y. (2017). Berbamine exerts anti-inflammatory effects via inhibition of NF-KB and MAPK signaling pathways. Cell. Physiol. Biochem. 41, 2307-2318. doi: 10.1159/0004 75650

Jung, D., and Bhattacharyya, N. (2012). Association of hearing loss with decreased employment and income among adults in the united states. Ann. Otol. Rhinol. Laryngol. 121, 771-775. doi: 10.1177/000348941212101201

Karasawa, T., Wang, Q., Fu, Y., Cohen, D. M., and Steyger, P. S. (2008). TRPV4 enhances the cellular uptake of aminoglycoside antibiotics. J. Cell Sci. 121, 2871-2879. doi: 10.1242/jcs.023705

Kenyon, E. J., Kirkwood, N. K., Kitcher, S. R. R., O’Reilly, M., Derudas, M., Cantillon, D. M., et al. (2017). Identification of ion-channel modulators that protect against aminoglycoside-induced hair cell death. JCI Insight 2:e96773. doi: 10.1172 /jci.insight.96773
Kirkwood, N. K., O’Reilly, M., Derudas, M., Kenyon, E. J., Huckvale, R., van Netten, S. M., et al. (2017). D-Tubocurarine and berbamine: alkaloids that are permeant blockers of the hair cell's mechano-electrical transducer channel and protect from aminoglycoside toxicity. Front. Cell. Neurosci. 11:262. doi: $10.3389 /$ fncel.2017.00262

Kitcher, S. R. R., Kirkwood, N. K., Camci, E. D., Wu, P., Gibson, R. M., Redila, V. A., et al. (2019). ORC-13661 protects sensory hair cells from aminoglycoside and cisplatin ototoxicity. JCI Insight 4:e126764. doi: $10.1172 /$ jci.insight. 126764

Koo, J.-W., Quintanilla-Dieck, L., Jiang, M., Liu, J., Urdang, Z. D., Allensworth, J. J., et al. (2015). Endotoxemia-mediated inflammation potentiates aminoglycoside-induced ototoxicity. Sci. Transl. Med. 7, 298ra118-298ra118. doi: 10.1126/scitranslmed.aac5546

Kruger, M., Boney, R., Ordoobadi, A. J., Sommers, T. F., Trapani, J. G., and Coffin, A. B. (2016). Natural bizbenzoquinoline derivatives protect zebrafish lateral line sensory hair cells from aminoglycoside toxicity. Front. Cell. Neurosci. 10:83. doi: 10.3389/fncel.2016.00083

Liang, Y., He, X., Li, X., Zhang, X., Zhang, X., Zhang, L., et al. (2016). 4chlorbenzoyl berbamine, a novel derivative of the natural product berbamine, potently inhibits the growth of human myeloma cells by modulating the NF-kB and JNK signalling pathways. Cancer Invest. 34, 496-505. doi: $10.1080 / 07357907.2016 .1235709$

Lukasz, D., and Kindt, K. S. (2018). In vivo calcium imaging of lateral-line hair cells in larval zebrafish. J. Vis. Exp. 141:10.3791/58794. doi: 10.3791/58794

Majumder, P., Moore, P. A., Richardson, G. P., and Gale, J. E. (2017). Protecting mammalian hair cells from aminoglycoside-toxicity: assessing phenoxybenzamine's potential. Front. Cell. Neurosci. 11:94. doi: 10.3389/fncel. 2017.00094

Marcotti, W., van Netten, S. M., and Kros, C. J. J. (2005). The aminoglycoside antibiotic dihydrostreptomycin rapidly enters mouse outer hair cells through the mechano-electrical transducer channels. J. Physiol. 567, 505-521. doi: 10.1113/jphysiol.2005.085951

Medeiros, M. A. A., Pinho, J. F., De-Lira, D. P., Barbosa-Filho, J. M., Araújo, D. A. M., Cortes, S. F., et al. (2011). Curine, a bisbenzylisoquinoline alkaloid, blocks L-type $\mathrm{Ca}^{2+}$ channels and decreases intracellular $\mathrm{Ca}^{2+}$ transients in A7r5 cells. Eur. J. Pharmacol. 669, 100-107. doi: 10.1016/j.ejphar. 2011.07.044

Mick, P., Kawachi, I., and Lin, F. R. (2014). The association between hearing loss and social isolation in older adults. Otolaryngol. Head Neck Surg. 150, 378-384. doi: 10.1177/0194599813518021

Murakami, S. L., Cunningham, L. L., Werner, L. A., Bauer, E., Pujol, R., Raible, D. W., et al. (2003). Developmental differences in susceptibility to neomycin-induced hair cell death in the lateral line neuromasts of zebrafish (danio rerio). Hear. Res. 186, 47-56. doi: 10.1016/s0378-5955(03)00259-4

Myrdal, S. E., and Steyger, P. S. (2005). TRPV1 regulators mediate gentamicin penetration of cultured kidney cells. Hear. Res. 204, 170-182. doi: 10.1016/j. heares.2005.02.005

Neveux, S., Smith, N. K., Roche, A., Blough, B. E., Pathmasiri, W., and Coffin, A. B. (2016). Natural compounds as occult ototoxins? Ginkgo biloba flavonoids moderately damage lateral line hair cells. J. Assoc. Res. Otolaryngol. 18, 275-289. doi: 10.1007/s10162-016-0604-6

Nyberg, S., Abbott, N. J., Shi, X., Steyger, P. S., and Dabdoub, A. (2019). Delivery of therapeutics to the inner ear: the challenge of the blood-labyrinth barrier. Sci. Transl. Med. 11:eaao0935. doi: 10.1126/scitranslmed.aao0935

O’Reilly, M., Kirkwood, N. K., Kenyon, E. J., Huckvale, R., Cantillon, D. M., Waddell, S. J., et al. (2019). Design, synthesis and biological evaluation of a new series of carvedilol derivatives that protect sensory hair cells from aminoglycoside-induced damage by blocking the mechanoelectrical transducer channel. J. Med. Chem. 62, 5312-5329. doi: 10.1021/acs.jmedchem.8b 01325

O'Sullivan, M. E., Perez, A., Lin, R., Sajjadi, A., Ricci, A. J., and Cheng, A. G. (2017). Towards the prevention of aminoglycoside-related hearing loss. Front. Cell. Neurosci. 11:325. doi: 10.3389/fncel.2017.00325

Ou, H. C., Cunningham, L. L., Francis, S. P., Brandon, C. S., Simon, J. A., Raible, D. W., et al. (2009). Identification of FDA-approved drugs and bioactives that protect hair cells in the zebrafish (danio rerio) lateral line and mouse (mus musculus) utricle. J. Assoc. Res. Otolaryngol. 10, 191-203. doi: 10.1007/s10162-009-0158-y 
Ou, H. C., Keating, S., Wu, P., Simon, J. A., Raible, D. W., and Rubel, E. W. (2012). Quinoline ring derivatives protect against aminoglycoside-induced hair cell death in the zebrafish lateral line. J. Assoc. Res. Otolaryngol. 13, 759-770. doi: 10.1007/s10162-012-0353-0

Ou, H. C., Santos, F., Raible, D. W., Simon, J. A., and Rubel, E. W. (2010). Drug screening for hearing loss: using the zebrafish lateral line to screen for drugs that prevent and cause hearing loss. Drug Discov. Today 15, 265-271. doi: 10.1016/j.drudis.2010.01.001

Owens, K. N., Coffin, A. B., Hong, L. S., O'Connell Bennett, K., Rubel, E. W., and Raible, D. W. (2009). Response of mechanosensory hair cells of the zebrafish lateral line to aminoglycosides reveals distinct cell death pathways. Hear. Res. 253, 32-41. doi: 10.1016/j.heares.2009.03.001

Owens, K. N., Santos, F., Roberts, B., Linbo, T., Coffin, A. B., Knisely, A. J., et al. (2008). Identification of genetic and chemical modulators of zebrafish mechanosensory hair cell death. PLoS Genet. 4:e1000020. doi: 10.1371/journal. pgen. 1000020

Perrey, D. A., German, N. A., Gilmour, B. P., Li, J.-X., Harris, D. L., Thomas, B. F., et al. (2013). Substituted tetrahydroisoquinolines as selective antagonists for the orexin 1 receptor. J. Med. Chem. 56, 6901-6916. doi: 10.1021/jm4 $00720 \mathrm{~h}$

Portmann, M., Darrouzet, J., and Coste, C. H. (1974). Distribution within the cochlea of dihydrostreptomycin injected into the circulation: an autoradiographic and electron microscopic study. Arch. Otolaryngol. 100, 473-475. doi: 10.1001/archotol.1974.00780040487014

Raible, D. W., and Kruse, G. J. (2000). Organization of the lateral line system in embryonic zebrafish. J. Comp. Neurol. 421, 189-198. doi: 10.1002/(sici)10969861(20000529)421:2<189::aid-cne5>3.0.co;2-k

Raviv, D., Dror, A. A., and Avraham, K. B. (2010). Hearing loss: a common disorder caused by many rare alleles. Ann. N Y Acad. Sci. 1214, 168-179. doi: 10.1111/j.1749-6632.2010.05868.x

Ren, Y., Lu, L., Guo, T. B., Qiu, J., Yang, Y., Liu, A., et al. (2008). Novel immunomodulatory properties of berbamine through selective down-regulation of STAT4 and action of ifn-gamma in experimental autoimmune encephalomyelitis. J. Immunol. 181, 1491-1498. doi: 10. 4049/jimmunol.181.2.1491

Santos, F., MacDonald, G., Rubel, E. W., and Raible, D. W. (2006). Lateral line hair cell maturation is a determinant of aminoglycoside susceptibility in zebrafish (Danio Rerio). Hear. Res. 213, 25-33. doi: 10.1016/j.heares.2005.12.009

Schulz, M., and Schmoldt, A. (2003). Therapeutic and toxic blood concentrations of more than 800 drugs and other xenobiotics. Pharmazie 58, 447-474. doi: 10.1016/bs.alkal.2018.11.002

Stepanyan, R. S., Indzhykulian, A. A., Vélez-Ortega, C. A., Boger, E. T., Steyger, P. S., Friedman, T. B., et al. (2011). TRPA1-Mediated Accumulation of Aminoglycosides in Mouse Cochlear Outer Hair Cells. J. Assoc. Res. Otolaryngol. 12, 729-740. doi: 10.1007/s10162-011-0288-x
Steyger, P. S., Peters, S. L., Rehling, J., Hordichok, A., and Dai, C. F. (2003). Uptake of gentamicin by bullfrog saccular hair cells in vitro. J. Assoc. Res. Otolaryngol. 4, 565-578. doi: 10.1007/s10162-003-4002-5

Teh, B. S., Seow, W. K., Li, S. Y., and Thong, Y. H. (1990). Inhibition of prostaglandin and leukotriene generation by the plant alkaloids tetrandrine and berbamine. Int. J. Immunopharmacol. 12, 321-326. doi: 10.1016/01920561(90)90088-5

Teles, M. M. R. S., Vieira Pinheiro, A. A., Da Silva Dias, C., Tavares, J. F., Barbosa Filho, J. M., and Leitão Da Cunha, E. V. (2019). The alkaloids: chemistry and biology. Alkaloids Chem. Biol. 82, 147-304. doi: 10.1016/bs.alkal.2018. 11.002

Thomas, A. J., Wu, P., Raible, D. W., Rubel, E. W., Simon, J. A., and Ou, H. C. (2015). Identification of small molecule inhibitors of cisplatin-induced hair cell death: results of a 10,000 compound screen in the zebrafish lateral line. Otol. Neurotol. 36, 519-525. doi: 10.1097/mao.0000000000000487

Tian, Y., and Zheng, J. (2017). Chapter six - metabolic activation and toxicities of bis-benzylisoquinoline alkaloids. Adv. Mol. Toxicol. 11, 241-272. doi: 10.1016/B978-0-12-812522-9.00006-3

Ton, C., and Parng, C. (2005). The use of zebrafish for assessing ototoxic and otoprotective agents. Hear. Res. 208, 79-88. doi: 10.1016/j.heares.2005.05.005

Vlasits, A. L., Simon, J. A., Raible, D. W., Rubel, E. W., and Owens, K. N. (2012). Screen of FDA-approved drug library reveals compounds that protect hair cells from aminoglycosides and cisplatin. Hear. Res. 294, 153-165. doi: 10.1016/j. heares.2012.08.002

Westerfield, M. (2000). The Zebrafish Book. A Guide for the Laboratory Use of Zebrafish (Danio Rerio). 4th Edn. Univsity of Oregon Press: Eugenecpubn.

Wiedenhoft, H., Hayashi, L., and Coffin, A. B. (2017). PI3K and inhibitor of apoptosis proteins modulate gentamicin-induced hair cell death in the zebrafish lateral line. Front. Cell. Neurosci. 11:326. doi: 10.3389/fncel.2017. 00326

Zhang, Q., Li, S., Wong, H.-T. C., He, X. J., Beirl, A., Petralia, R. S., et al. (2018). Synaptically silent sensory hair cells in zebrafish are recruited after damage. Nat. Commun. 9:1388. doi: 10.1038/s41467-018-03806-8

Conflict of Interest: The authors declare that the research was conducted in the absence of any commercial or financial relationships that could be construed as a potential conflict of interest.

Copyright (c) 2020 Hudson, Lockard, Namjoshi, Wilson, Kindt, Blough and Coffin. This is an open-access article distributed under the terms of the Creative Commons Attribution License (CC BY). The use, distribution or reproduction in other forums is permitted, provided the original author(s) and the copyright owner(s) are credited and that the original publication in this journal is cited, in accordance with accepted academic practice. No use, distribution or reproduction is permitted which does not comply with these terms. 